\title{
RISK MINIMIZATION FOR GAME OPTIONS IN MARKETS IMPOSING MINIMAL TRANSACTION COSTS
}

\author{
YAN DOLINSKY AND YURI KIFER \\ HEBREW UNIVERSITY OF JERUSALEM
}

\begin{abstract}
We study partial hedging for game options in markets with transaction costs bounded from below. More precisely, we assume that the investor's transaction costs for each trade are the maximum between proportional transaction costs and a fixed transaction costs. We prove that in the continuous time Black-Scholes (BS) model, there exists a trading strategy which minimizes the shortfall risk. Furthermore, we use binomial models in order to provide numerical schemes for the calculation of the shortfall risk and the corresponding optimal portfolio in the BS model.
\end{abstract}

\section{INTRODUCTION}

A game contingent claim (GCC) or game option, which was introduced in [14, is defined as a contract between the seller and the buyer of the option such that both have the right to exercise it at any time up to a maturity date (horizon) $T$. If the buyer exercises the contract at time $t$ then he receives the payment $Y_{t}$, but if the seller exercises (cancels) the contract before the buyer then the latter receives $X_{t}$. The difference $\Delta_{t}=X_{t}-Y_{t}$ is the penalty which the seller pays to the buyer for the contract cancellation. In short, if the seller will exercise at a stopping time $\sigma \leq T$ and the buyer at a stopping time $\tau \leq T$ then the former pays to the latter the amount $H(\sigma, \tau)$ where $H(\sigma, \tau)=X_{\sigma} \mathbb{I}_{\sigma<\tau}+Y_{\tau} \mathbb{I}_{\tau \leq \sigma}$ and we set $\mathbb{I}_{Q}=1$ if an event $Q$ occurs and $\mathbb{I}_{Q}=0$ if not.

A hedge (for the seller) against a GCC is defined as a pair $(\pi, \sigma)$ that consists of a self financing strategy $\pi$ and a stopping time $\sigma$ which is the cancellation time for the seller whom we also call an investor. For more details on game options see [15.

In this paper we study hedging with transaction costs of the following form. If the investor makes a small trade then he pays a fixed transaction costs, and if the investor makes a large trade he pays proportional transaction costs. Formally, for buying (or selling) $\beta \neq 0$ stocks the transaction costs are given by $\max (\delta, \mu|\beta| S)$ where $\delta>0,0<\mu<1$ are constants and $S$ is the stock price at the moment of the trade. The investor's total transaction costs should be finite, hence in our setup the investor can trade only a finite (random) number of times. Although this type of transaction costs is very natural and widespread it was not studied much so far.

In 4 it was proved that super-replication of game options under proportional transaction costs is expensive and leads to trivial (buy-and-hold) strategies. Since

Date: August 10, 2018.

2010 Mathematics Subject Classification. 91G10, 91G20, 60F15, 60G40, 60G44.

Key words and phrases. game options, transaction costs, hedging with friction, risk minimization. 
the friction in our setup is larger than the friction in the proportional costs setup, then similar results hold true for our case as well. Therefore, with the presence of transaction costs, it is reasonable to assume that the seller's (investor's) initial capital is less than the superhedging price, and so hedging with risk comes into the picture. We deal with a certain type of risk called the shortfall risk, which is the maximal expectation with respect to the buyer exercise times of the shortfall. For the definition of the shortfall risk measure for game options see [5] and $[\underline{6}$.

There are several papers which study shortfall minimization with friction (see for instance, [3, 8], 9], 11] and [12]). All of these papers considered the proportional transaction costs setup (for European and American options) for which they proved an existence of an optimal hedge.

In real market conditions transaction costs generally contain a fixed component, i.e. the transaction costs are bounded from below by a positive constant. Many authors considered utility maximization under transaction costs with a fixed component. For details see ([1, 7], 16, 19, [20 and 21]). However for partial hedging of derivative securities this setup was not studied before.

In this paper we consider a game option in the BS model with continuous path dependent payoffs. Our first result says that for our type of frictions there is an optimal hedge. In general, the problem of the existence of an optimal hedge for the shortfall risk measure in a game options setup is much more complicated than for European and American options. The reason is that for game options the shortfall risk measure fails to be a convex functional of the portfolio strategy, and so the compactness principle which relies on the Komlos lemma can not applied here. This is the principle which applied for European and American options in all of the mentioned above papers. For the type of transaction costs considered here convexity arguments do not work for any type of options, and so our result is new even for American options (as a special case of game options) though in this case the proof is simpler. For game options in a setup without friction or with friction smaller than we consider in this paper, the existence of a shortfall minimization hedge is an open question.

Our approach is to establish the continuity of the shortfall risk function and to reduce the optimization problem to a Dynkin game with continuous payoffs. Then we apply Dynkin games theory in Brownian setup and use the fact that the set of all permissible trades is compact.

Next, we deal with the computational aspect of shortfall risk minimization. We employ an appropriate sequence of binomial models in order to approximate the shortfall risk and to construct almost optimal portfolios in the BS model. For Lipschitz continuous (may be path dependent) payoffs we obtain two sided error estimates for the binomial approximations. So far, shortfall risk approximations for game options were considered only in the frictionless setup (see [6]), where we obtained only one sided error estimates. In general, in the presence of friction which is no less than proportional transaction costs, we can find a uniform bound for the growth of admissible portfolios. This bound is essential for establishing the error estimates.

For game options in binomial models the shortfall risk and the corresponding optimal portfolio can be calculated by a dynamical programming algorithm. Thus, these approximation theorems provide an efficient tool for numerical calculation of the shortfall risk and the corresponding optimal hedge in the BS model. 
Main results of this paper are formulated in the next section. In Section 3 we prove the existence of an optimal hedge (Theorem 2.1). In Section 4 we prove the approximation results (Theorem 2.2). The proof of both Theorems 2.1 and 2.2 rely on some regularity properties of the shortfall risk whose proofs we postpone till Section 5 .

\section{Preliminaries and Main Results}

Consider a complete probability space $(\Omega, \mathcal{F}, \mathbb{P})$ together with a standard onedimensional Brownian motion $\left\{W_{t}\right\}_{t=0}^{\infty}$, and the filtration $\mathcal{F}_{t}=\sigma\left\{W_{s} \mid s \leq t\right\}$ completed by the null sets. Our BS financial market consists of a safe asset $B$ used as numeraire, hence $B \equiv 1$, and of a risky asset $S$ whose value at time $t$ is given by

$$
S_{t}^{(s)}=s \exp \left(\kappa W_{t}+\left(\vartheta-\kappa^{2} / 2\right) t\right), \quad s>0, \quad t \geq 0
$$

where $\kappa>0$ is called volatility and $\vartheta \in \mathbb{R}$ is another constant. It is well known that for the BS model there exists a unique probability measure $\mathbb{Q} \sim \mathbb{P}$ such that the stock price process $S^{(s)}$ is a $\mathbb{Q}$ martingale. Using standard arguments it follows that the restriction of the probability measure $\mathbb{Q}$ to the $\sigma$-algebra $\mathcal{F}_{t}$ satisfies

$$
Z_{t}:=\frac{d \mathbb{Q}}{d \mathbb{P}} \mid \mathcal{F}_{t}=\exp \left(-\frac{\vartheta}{\kappa} W_{t}-\frac{1}{2}\left(\frac{\vartheta}{\kappa}\right)^{2} t\right) .
$$

Next, let $T<\infty$ and let $C[0, T]$ be the space of all continuous functions $f$ : $[0, T] \rightarrow \mathbb{R}$ equipped with the uniform topology. Denote by $C_{++}[0, T] \subset C[0, T]$ the subset of all strictly positive functions. Let $F, G: C[0, T] \rightarrow C[0, T]$ be continuous progressively measurable functions which means that for any $t \in[0, T]$ and $x, y \in$ $C[0, T], G(x)_{[0, t]}=G(y)_{[0, t]}$ and $F(x)_{[0, t]}=F(y)_{[0, t]}$ if $x_{[0, t]}=y_{[0, t]}$. We assume that $F \leq G$ and there exist constants $C, p>0$ for which

$$
\|F(x)\|+\|G(x)\| \leq C\left(1+\|x\|^{p}\right)
$$

where $\|\cdot\|$ denotes the sup norm on the space $C[0, T]$. Consider a game option with maturity date $T$ and continuous payoffs which are given by

$$
Y_{t}^{(s)}=\left[F\left(S^{(s)}\right)\right](t) \leq\left[G\left(S^{(s)}\right)\right](t)=X_{t}^{(s)} .
$$

In our model, purchase and sale of the risky asset are subject to transactions costs which are the maximum of a constant fee and a proportional transaction cost. Namely, if the investor buys (or sells) $\beta$ stocks then his transaction costs are given by

$$
g(\beta, S):=\max (\delta, \mu|\beta| S) \mathbb{I}_{\beta \neq 0}
$$

where $\delta>0,0<\mu<1$ are constants and $S$ is the stock price at the moment of trade. Presence of this minimal transaction cost yields that in order to avoid infinite transaction costs portfolios can only be rebalanced finitely many (but a random number of) times.

Next, we define hedging and shortfall risk in the above setup. A (self financing) trading strategy with an initial position $(z, y)$ is a triple $\pi=(z, y, \gamma)$ where $z$ is the cash value of the portfolio at the initial time, $y$ is the number of stocks at this moment, and $\gamma=\left\{\gamma_{t}\right\}_{t=0}^{T}$ is an adapted, left continuous, pure jump process with finite (random) number of jumps and initial value $\gamma_{0}=y$. The random variable $\gamma_{t}$ denotes the number of shares in the portfolio $\pi$ at time $t$ before any change is made at this time (which is the reason why we assume that the process $\gamma$ is left 
continuous). Observe that at time 0 the investor has the value $z+g(y, s)-y s$ on his savings account. Thus the portfolio (cash) value of a trading strategy $\pi$ at time $t$ is given by

$$
V_{t}^{\pi}=z+\int_{0}^{t} \gamma_{u} d S_{u}^{(s)}+g(y, s)-g\left(\gamma_{t}, S_{t}^{(s)}\right)-\sum_{u \in[0, t)} g\left(\gamma_{u+}-\gamma_{u}, S_{u}^{(s)}\right),
$$

where in the last sum there is only finitely many terms which are not equal to zero. A portfolio $\pi$ will be called admissible if $V_{t}^{\pi} \geq 0$ for any $t$. A hedge consists of a trading strategy and a cancellation time. Thus, formally a hedge with initial position $(z, y)$ is a pair $(\pi, \sigma)$ such that $\pi$ is an admissible portfolio and $\sigma \leq T$ is a stopping time (with respect to the Brownian filtration). From (2.4) it follows that for an admissible portfolio $\pi$ the stochastic process $V_{t}^{\pi}, t \geq 0$ is a super-martingale with respect to the martingale measure $\mathbb{Q}$. The set of all hedges with initial position $(z, y) \in \mathbb{R}_{+} \times \mathbb{R}$ will be denoted by $\mathcal{A}(T, s, z, y)$. The set of all hedges will be denoted by $\mathcal{A}(T, s)$, where $s$ is the initial stock price and $T$ is the maturity date.

Next, we define the shortfall risk. Denote by $\mathcal{T}_{T}$ the set of all stopping times less or equal than $T$. For a hedge $(\pi, \sigma)$ the shortfall risk is defined by

$$
\mathcal{R}(T, s, \pi, \sigma)=\sup _{\tau \in \mathcal{T}_{T}} \mathbb{E}_{\mathbb{P}}\left(X_{\sigma}^{(s)} \mathbb{I}_{\sigma<\tau}+Y_{\tau}^{(s)} \mathbb{I}_{\tau \leq \sigma}-V_{\sigma \wedge \tau}^{\pi}\right)^{+},
$$

which is the maximal possible expectation with respect to the probability measure $\mathbb{P}$ of the shortfall. The shortfall risk for an initial position $(z, y)$ is given by

$$
R(T, s, z, y)=\inf _{(\pi, \sigma) \in \mathcal{A}(T, s, z, y)} \mathcal{R}(T, s, \pi, \sigma) .
$$

The following theorem says that for a given initial position $(z, y)$ there exists a hedge which minimizes the shortfall risk.

Theorem 2.1. Let $(z, y) \in \mathbb{R}_{+} \times \mathbb{R}$ be an initial position. There exists a hedge (may be not unique) $(\hat{\pi}, \hat{\sigma}) \in \mathcal{A}(T, s, z, y)$ such that $\mathcal{R}(T, s, \hat{\pi}, \hat{\sigma})=R(T, s, z, y)$.

Next, we approximate the shortfall risk in the Black-Scholes model by a sequence of binomial models. In order to obtain error estimates we will assume that the functions $F, G$ can be extended to the space $D[0, T]$ (of all càdlàg functions on the interval $[0, T]$ ) and satisfy the following Lipschitz condition. There exists a constant $L$ such that for any $0 \leq t_{1}<t_{2} \leq T$ and $x, y \in D[0, T]$,

$$
\begin{gathered}
\|F(y)-F(x)\|+\|G(y)-G(x)\| \leq L|| y-x \| \text { and } \\
\left|F(x)\left(t_{2}\right)-F(x)\left(t_{1}\right)\right|+\left|G(x)\left(t_{2}\right)-G(x)\left(t_{1}\right)\right| \leq \\
L\left(\left(t_{2}-t_{1}\right)(1+\|x\|)+\sup _{t_{1} \leq u \leq t_{2}}|x(u)-x(t)|\right) .
\end{gathered}
$$

For any $n$ consider a binomial model which consists of a savings account $\equiv 1$, and of a piecewise constant risky asset $\left\{S_{t}^{n, s}\right\}_{t=0}^{T}$ given by

$$
S_{t}^{n, s}=s \exp \left(\kappa \sqrt{\frac{T}{n}} \sum_{i=1}^{[n t / T]} \xi_{i}\right), t \in[0, T]
$$

where [.] denotes the integer part and $\xi_{1}, \xi_{2}, \ldots$ are i.i.d. random variables taking values 1 and -1 with probabilities $p^{(n)}=\left(\exp \left(\left(\kappa-\frac{2 \vartheta}{\kappa}\right) \sqrt{\frac{T}{n}}\right)+1\right)^{-1}$ and $1-p^{(n)}=$ 
$\left(\exp \left(\left(\frac{2 \vartheta}{\kappa}-\kappa\right) \sqrt{\frac{T}{n}}\right)+1\right)^{-1}$, respectively. Let $\mathbb{P}_{n}$ be the corresponding probability measure and let $\mathcal{F}_{t}^{(n)}=\sigma\left\{S_{u}^{n, s}: u \leq t\right\}$ be the filtration which is generated by $S^{n, s}$.

Denote by $\mathcal{A}^{(n)}(T, s, z, y)$ the set of all hedges with an initial position $(z, y)$. The definition of a hedge is done in analogous way to the Black-Scholes model, just replace the Brownian filtration by $\mathcal{F}^{(n)}$ and $S^{(s)}$ by $S^{n, s}$ in (2.4).

We introduce game options with the piecewise constant payoffs

$$
Y_{t}^{n, s}=\left[F\left(S^{n, s}\right)\right]([n t / T] T / n) \leq X_{t}^{n, s}=\left[G\left(S^{n, s}\right)\right]([n t / T] T / n), \quad t \in[0, T] .
$$

Define the shortfall risk

$$
\mathcal{R}_{n}(T, s, \pi, \sigma)=\sup _{\tau \in \mathcal{T}_{T}^{(n)}} \mathbb{E}_{\mathbb{P}_{n}}\left(X_{\sigma}^{n, s} \mathbb{I}_{\sigma<\tau}+Y_{\tau}^{n, s} \mathbb{I}_{\tau \leq \sigma}-V_{\sigma \wedge \tau}^{\pi}\right)^{+},
$$

where $\mathcal{T}_{T}^{(n)}$ is the set of all stopping times less than $T$. The shortfall risk for an initial position $(z, y)$ is given by $R_{n}(T, s, z, y)=\inf _{(\pi, \sigma) \in \mathcal{A}^{(n)}(T, s, z, y)} \mathcal{R}_{n}(T, s, \pi, \sigma)$.

Next, we introduce a simple form of Skorokhod embedding which allows to consider the above binomial markets and the BS model on the same probability space. Set $W_{t}^{*}=\frac{\ln S_{t}^{(s)}-\ln s}{\kappa}, t \geq 0$, and for any $n \in \mathbb{N}$ define recursively $\theta_{0}^{(n)}=0, \theta_{k+1}^{(n)}=$ $\inf \left\{t>\theta_{k}^{(n)}:\left|W_{t}^{*}-W_{\theta_{k}^{(n)}}^{*}\right|=\sqrt{\frac{T}{n}}\right\}$. Observe (see [6]) that for any $k, W_{\theta_{k+1}^{(n)}}^{*}-W_{\theta_{k}^{(n)}}^{*}$ is independent of $\mathcal{F}_{\theta_{k}^{(n)}}$ and takes on the values $\sqrt{\frac{T}{n}}$ and $-\sqrt{\frac{T}{n}}$, with probabilities $p^{(n)}$ and $1-p^{(n)}$, respectively. For any $n$, define the map $\Pi_{n}: L^{\infty}\left(\mathcal{F}_{T}^{(n)}, \mathbb{P}_{n}\right) \rightarrow$ $L^{\infty}\left(\mathcal{F}_{\theta_{n}^{(n)}}, \mathbb{P}\right)$ by $\Pi_{n}(U)=\tilde{U}$ so that if $U=f\left(\sqrt{\frac{T}{n}} \xi_{1}, \ldots, \sqrt{\frac{T}{n}} \xi_{n}\right)$ for a function $f$ on $\left\{\sqrt{\frac{T}{n}},-\sqrt{\frac{T}{n}}\right\}^{n}$ then $\tilde{U}=f\left(W_{\theta_{1}^{(n)}}^{*}, W_{\theta_{2}^{(n)}}^{*}-W_{\theta_{1}^{(n)}}^{*}, \ldots, W_{\theta_{n}^{(n)}}^{*}-W_{\theta_{n-1}^{(n)}}^{*}\right)$.

The map $\Pi_{n}$ allows to lift hedges from the binomial models to the BS model. For an initial position $(z, y)$ denote by $\mathcal{A}^{W, n}(s, z, y)$ the set of all admissible self financing strategies which are managed on the set $\left\{0, \theta_{1}^{(n)}, \ldots, \theta_{n}^{(n)}\right\}$ such that after the time $\theta_{n}^{(n)}$ the number of stocks in the portfolio is 0. Namely, $\pi=\left(z, y,\left\{\tilde{\gamma}_{t}\right\}_{t=0}^{\infty}\right) \in$ $\mathcal{A}^{W, n}(s, z, y)$ if $\tilde{\gamma}_{t}$ is constant on the interval $\left(\theta_{k}^{(n)}, \theta_{k+1}^{(n)}\right], k<n$ and $\tilde{\gamma}_{t} \equiv 0$ on $\left(\theta_{n}^{(n)}, \infty\right)$. The portfolio value is given by (2.4). Define the lifting $\Psi_{n}: \mathcal{A}^{(n)}(T, s, z, y) \rightarrow$ $\mathcal{A}^{W, n}(s, z, y) \times \mathcal{T}_{T}, \Psi_{n}(\pi, \sigma)=(\tilde{\pi}, \tilde{\sigma})$ as follows. Let $\pi=(z, y, \gamma)$. Then $\tilde{\pi}=(z, y, \tilde{\gamma})$ where

$$
\tilde{\gamma}_{t}=y \mathbb{I}_{t=0}+\sum_{i=0}^{n-1} \mathbb{I}_{\theta_{i}^{(n)}<t \leq \theta_{i+1}^{(n)}} \Pi_{n}\left(\gamma_{(i+1) T / n}\right) .
$$

Similar arguments as in 3 , (see Section 2 there) yield that

$$
V_{\theta_{k}^{(n)}}^{\tilde{\pi}}=\Pi_{n}\left(V_{k T / n}^{\pi}\right) \quad k=0,1, \ldots, n
$$

and $V_{t}^{\tilde{\pi}} \geq 0, t \geq 0$. Furthermore, the portfolio value $V_{t}^{\tilde{\pi}}$ is constant after $\theta_{n}^{(n)}$. Observe that if we restrict the portfolio $\tilde{\pi}$ to the interval $[0, T]$ we get an element in $\mathcal{A}(T, s, z, y)$. Next, we define $\tilde{\sigma} \in \mathcal{T}_{T}$ by

$$
\tilde{\sigma}=T \wedge \theta_{\Pi_{n}(\sigma)}^{(n)} \text { if } \Pi_{n}(\sigma)<n \text { and } \tilde{\sigma}=T \text { if } \Pi_{n}(\sigma)=n .
$$

The following theorem says that the shortfall risk in the BS model can be approximated by the shortfall risks in the binomial models defined above. Furthermore, by 
lifting the optimal hedges in the binomial models we get "almost" optimal hedges in the BS model.

Theorem 2.2. Let $(z, y) \in \mathbb{R}_{++} \times \mathbb{R}$ be an initial position. There exists a constant $C>0$ such that for any $n \in \mathbb{N}$

$$
\left|R(T, s, z, y)-R_{n}(T, s, z, y)\right| \leq C n^{-1 / 4}(\ln n)^{3 / 4} .
$$

Furthermore, let $\left(\pi_{n}, \sigma_{n}\right) \in \mathcal{A}^{(n)}(T, s, z, y)$ be an optimal hedge, i.e. $\mathcal{R}_{n}\left(T, s, \pi_{n}, \sigma_{n}\right)=$ $R_{n}(T, s, z, y)$. Then for the hedges $\left(\tilde{\pi}_{n}, \tilde{\sigma}_{n}\right)=\Psi_{n}\left(\pi_{n}, \sigma_{n}\right), n \in \mathbb{N}$, we have

$$
\mathcal{R}\left(T, s, \tilde{\pi}_{n}, \tilde{\sigma}_{n}\right) \leq R(T, s, z, y)+C n^{-1 / 4}(\ln n)^{3 / 4},
$$

where in the above right hand side we take the restriction of $\tilde{\pi}_{n}$ to the interval [0,T].

Remark 2.3. Theorems 2.1 2.2 can be extended to the case where the constant component $\delta$ becomes a Lipschitz continuous function of time $\delta(t)$. This makes sense since then we can consider constant minimal transaction cost $\delta$ with respect to the original currency where measured by numeraire $\delta(t)=\delta e^{r t}$ with $r$ being the interest rate. In this case the portfolio value process may no longer be a super-martingale under the martingale measure $\mathbb{Q}$. The super-martingale property is used in Lemma 5.3. However, by applying the growth results obtained in Lemma 4.1 we can still prove the statement of Lemma 5.3. In this case the proofs of both Theorems 2.1 and 2.2 will become more technical and somewhat unwieldy. The first reason for these complications is that in a setup where the constant component depends on time, the lifting of the hedges more involved and it requires truncation of the portfolio in order to keep the admissability condition. The second reason is that Lemma 4.3 requires a new proof, since the portfolio strategy is not a super-martingale. Both of these steps can be done using Lemma 4.1 but in order to simplify exposition we will deal here only with the case where $\delta$ is constant with respect to the numeraire.

\section{Proof of Theorem 2.1}

We start with some preparations. For any $\mathbf{T} \leq T$ and $v \in C_{++}[0, T]$ define the continuous stochastic process $\left\{S_{t}^{\mathbf{T}, v}\right\}_{t=0}^{T}$ by

$$
S_{t}^{\mathbf{T}, v}=v_{T-\mathbf{T}} \mathbb{I}_{t \leq T-\mathbf{T}}+S_{t+\mathbf{T}-T}^{\left(v_{T-\mathbf{T}}\right)} \mathbb{I}_{t>T-\mathbf{T}} .
$$

Namely, $S^{\mathbf{T}, v}$ coincides with $v$ on the interval $[0, T-\mathbf{T}]$ and $S_{t}^{\mathbf{T}, v}$ is a geometric Brownian motion for $t>T-\mathbf{T}$. Consider a cash settled game option with a maturity date $\mathbf{T}<\infty$ defined in a BS financial market which is described in Section 2. The payoffs are given by

$$
Y_{t}^{\mathbf{T}, v}=\left[F\left(S^{\mathbf{T}, v}\right)\right](t+T-\mathbf{T}) \text { and } X_{t}^{\mathbf{T}, v}=\left[G\left(S^{\mathbf{T}, v}\right)\right](t+T-\mathbf{T}) \quad t \in[0, \mathbf{T}] .
$$

Observe that the processes $X_{t}^{\mathbf{T}, v} \geq Y_{t}^{\mathbf{T}, v}, t \in[0, \mathbf{T}]$ are continuous and adapted. Furthermore, if $\mathbf{T}=T$ then $X_{t}^{\mathbf{T}, v}=X_{t}^{\left(v_{0}\right)}$ and $Y_{t}^{\mathbf{T}, v}=Y_{t}^{\left(v_{0}\right)}$ for $t \in[0, T]$.

Next, we define the shortfall risk for a maturity $\mathbf{T} \leq T$. The sets $\mathcal{A}(\mathbf{T}, s, z, y)$ and $\mathcal{T}_{\mathbf{T}}$ are defined as in Section 2 , just replace $T$ by $\mathbf{T}$. For $v \in C_{++}[0, T]$ and a hedge $(\pi, \sigma) \in \mathcal{A}\left(\mathbf{T}, v_{T-\mathbf{T}}, z, y\right)$ the shortfall risk is defined by

$$
\mathcal{R}(\mathbf{T}, v, \pi, \sigma)=\sup _{\tau \in \mathcal{T}_{\mathbf{T}}} \mathbb{E}_{\mathbb{P}}\left(X_{\sigma}^{\mathbf{T}, v} \mathbb{I}_{\sigma<\tau}+Y_{\tau}^{\mathbf{T}, v} \mathbb{I}_{\tau \leq \sigma}-V_{\sigma \wedge \tau}^{\pi}\right)^{+} .
$$


Similarly to above we set $R(\mathbf{T}, v, z, y)=\inf _{(\pi, \sigma) \in \mathcal{A}\left(\mathbf{T}, v_{T-\mathbf{T}}, z, y\right)} \mathcal{R}(\mathbf{T}, v, \pi, \sigma)$. Observe that $\mathcal{R}(\mathbf{T}, v, \cdot, \cdot \cdot)$ and $R(\mathbf{T}, v, \cdot, \cdot \cdot)$ depend only on $v_{[0, T-\mathbf{T}]}$. Furthermore for $\mathbf{T}=T$, if $v_{0}=s$ then $\mathcal{R}(T, v, \cdot, \cdot \cdot)=\mathcal{R}(T, s, \cdot, \cdot \cdot)$ and $R(T, v, \cdot, \cdot \cdot)=R(T, s, \cdot, \cdot \cdot)$.

Now, assume that at a given time a portfolio value is $z$, the number of stocks is $y$ and the stock price at this moment is $S$. If the investor buys $\beta \neq 0$ stocks then the new (cash settled) portfolio value will be

$$
h(S, z, y, \beta):=z+g(y, S)-g(y+\beta, S)-g(\beta, S) .
$$

For $\beta=0$ we define the function $h$ such that it will be continuous in $\beta=0$. Thus we set $h(S, z, y, 0)=z-\delta$. Let $\Gamma(S, z, y)$ be the set of all $\beta$ which satisfy $h(S, z, y, \beta) \geq 0$. It is clear that $\Gamma(S, z, y)$ is a compact set. Observe also that a portfolio strategy is admissible if and only if it consists of permissible trades. For $y \neq 0$ we have that $-y \in \Gamma(S, z, y)$, and so the set $\Gamma(S, z, y)$ is not empty. For $y=0$ the set $\Gamma(S, z, y)$ is empty if and only if $z<\delta$. Define,

$$
\hat{R}(\mathbf{T}, v, z, y)=\min \left(\left(X_{0}^{\mathbf{T}, v}-z\right)^{+}, \inf _{\beta \in \Gamma\left(v_{T-\mathbf{T}}, z, y\right)} R\left(\mathbf{T}, v, h\left(v_{T-\mathbf{T}}, z, y, \beta\right), \beta+y\right)\right)
$$

where the infimum over an empty set is $\infty$.

Next, let $s$ be the initial stock price and $(z, y)$ be the initial position of the investor. Set

$$
\mathbf{V}_{t}^{s, z, y}=z+g(y, s)+y\left(S_{t}^{(s)}-s\right)-g\left(y, S_{t}^{(s)}\right), \quad t \geq 0 .
$$

Observe that $\mathbf{V}_{t}^{s, z, y}$ is the portfolio value at time $t$ of the investor who did not trade until this time.

The following result is the first step in the proof of Theorem 2.1.

Lemma 3.1. Let $(\mathbf{T}, v, z, y) \in[0, T] \times C_{++}[0, T] \times \mathbb{R}_{+} \times \mathbb{R}$ and let $s=v_{T-\mathbf{T}}$ be the initial stock price. Define the stopping time $\Theta=\mathbf{T} \wedge \inf \left\{t: \mathbf{V}_{t}^{s, z, y}<0\right\}$. Then,

$$
\begin{aligned}
& R(\mathbf{T}, v, z, y) \geq \inf _{\sigma \in \mathcal{T}_{\mathbf{T}}, \sigma \leq \Theta} \sup _{\tau \in \mathcal{T}_{\mathbf{T}}, \tau \leq \Theta} \mathbb{E}_{\mathbb{P}}\left(\mathbb{I}_{\tau \leq \sigma} \times\right. \\
& \left.\left(Y_{\tau}^{\mathbf{T}, v}-\mathbf{V}_{\tau}^{s, z, y}\right)^{+}+\mathbb{I}_{\sigma<\tau} \hat{R}\left(\mathbf{T}-\sigma, S^{\mathbf{T}, v}, \mathbf{V}_{\sigma}^{s, z, y}, y\right)\right) .
\end{aligned}
$$

Proof. Let $(\pi, \sigma) \in \mathcal{A}(\mathbf{T}, s, z, y)$. Set $\sigma_{1}=\sigma \wedge \min \left\{t: \gamma_{t} \neq \gamma_{t+}\right\}$ where $\pi=(z, y, \gamma)$. Clearly $\sigma_{1} \in \mathcal{T}_{\mathbf{T}}$ is a stopping time. Introduce the stochastic process

$$
U_{t}=e s s \sup _{\tau \geq t, \tau \in \mathcal{T}_{\mathbf{T}}} \mathbb{E}_{\mathbb{P}}\left(\left(\mathbb{I}_{\tau \leq \sigma} Y_{\tau}^{\mathbf{T}, v}+\mathbb{I}_{\sigma<\tau} X_{\sigma}^{\mathbf{T}, v}-V_{\sigma \wedge \tau}^{\pi}\right)^{+} \mid \mathcal{F}_{t}\right), t \in[0, \mathbf{T}] .
$$

The stochastic process $\left\{\mathbb{I}_{t \leq \sigma} Y_{t}^{\mathbf{T}, v}+\mathbb{I}_{\sigma<t} X_{\sigma}^{\mathbf{T}, v}-V_{\sigma \wedge t}^{\pi}\right\}_{t=0}^{\mathbf{T}}$ is left continuous with right hand limits. Furthermore, this process is lower semi-continuous from the right. Thus from the general theory of optimal stopping (see [17] and the references there) it follows that $\left\{U_{t}\right\}_{t=0}^{\mathbf{T}}$ is a càdlàg process and for any stopping time $\rho \leq \mathbf{T}$,

$$
U_{\rho}=e s s \sup _{\tau \in \mathcal{T}_{\mathbf{T}}^{\rho}} \mathbb{E}_{\mathbb{P}}\left(\left(\mathbb{I}_{\tau \leq \sigma} Y_{\tau}^{\mathbf{T}, v}+\mathbb{I}_{\sigma<\tau} X_{\sigma}^{\mathbf{T}, v}-V_{\sigma \wedge \tau}^{\pi}\right)^{+} \mid \mathcal{F}_{\rho}\right)
$$

where $\mathcal{T}_{\mathbf{T}}^{\rho}$ is the set of all stopping times $\rho \leq \tau \leq \mathbf{T}$ which satisfy $\tau>\rho \mathbb{I}_{\rho<\mathbf{T}}$.

Clearly, there is no trade until the time $\sigma_{1}$. Thus $V_{t}^{\pi}=\mathbf{V}_{t}^{s, z, y}$ for $t \leq \sigma_{1}$. This together with the fact that $\sigma_{1} \leq \sigma$ and (3.3) (for $\rho=\sigma_{1}$ ) yields

$$
\mathcal{R}(\mathbf{T}, v, \pi, \sigma)=\sup _{\tau \in \mathcal{T}_{\mathbf{T}}} \mathbb{E}_{\mathbb{P}}\left(\mathbb{I}_{\sigma_{1}<\tau} U_{\sigma_{1}}+\mathbb{I}_{\tau \leq \sigma_{1}}\left(Y_{\tau}^{\mathbf{T}, v}-\mathbf{V}_{\tau}^{s, z, y}\right)^{+}\right) .
$$


From the Markov property of the Brownian motion it follows that

$$
U_{t} \geq R\left(\mathbf{T}-t, S^{\mathbf{T}, v}, V_{t}^{\pi}, \gamma_{t}\right) .
$$

Thus, by the continuity of $R$ (see Proposition 5.5) it follows that on the event $\sigma_{1}<\mathbf{T}$,

$$
\begin{gathered}
U_{\sigma_{1}}=\mathbb{I}_{\sigma_{1}=\sigma}\left(X_{\sigma_{1}}^{\mathbf{T}, v}-V_{\sigma_{1}}^{\pi}\right)^{+}+\mathbb{I}_{\sigma_{1}<\sigma} \lim _{t \downarrow \sigma_{1}} U_{t} \geq \\
\min \left(\left(X_{\sigma_{1}}^{\mathbf{T}, v}-V_{\sigma_{1}}^{\pi}\right)^{+}, R\left(\mathbf{T}-\sigma_{1}, S^{\mathbf{T}, v}, V_{\sigma_{1}+}^{\pi}, \gamma_{\sigma_{1}+}\right)\right) \geq \\
\hat{R}\left(\mathbf{T}-\sigma_{1}, S^{\mathbf{T}, v}, V_{\sigma_{1}}^{\pi}, \gamma_{\sigma_{1}}\right)=\hat{R}\left(\mathbf{T}-\sigma_{1}, S^{\mathbf{T}, v}, \mathbf{V}_{\sigma_{1}}^{s, z, y}, y\right) .
\end{gathered}
$$

From (3.4)-(3.5) and the inequality $\sigma_{1} \leq \Theta$ we get

$$
\begin{gathered}
\mathcal{R}(\mathbf{T}, v, \pi, \sigma) \geq \inf _{\sigma \in \mathcal{T}_{\mathbf{T}}, \sigma \leq \Theta} \sup _{\tau \in \mathcal{T}_{\mathbf{T}, \tau} \leq \Theta} \mathbb{E}_{\mathbb{P}}\left(\mathbb{I}_{\tau \leq \sigma} \times\right. \\
\left.\left(Y_{\tau}^{\mathbf{T}, v}-\mathbf{V}_{\tau}^{s, z, y}\right)^{+}+\mathbb{I}_{\sigma<\tau} \hat{R}\left(\mathbf{T}-\sigma, S^{\mathbf{T}, v}, \mathbf{V}_{\sigma}^{s, z, y}, y\right)\right)
\end{gathered}
$$

and since $(\pi, \sigma)$ was arbitrary the proof is completed.

Next, we construct an optimal hedge and verify that it is indeed optimal. The verification will be done by showing that for the constructed hedge the left hand side and the right hand side of (3.6) are equal.

Let $(z, y)$ be an initial position. Set, $\hat{\sigma}_{0}=0, \hat{\gamma}_{0}=y, \hat{Z}_{0}=z$. For $k \geq 1$ define the random time $\Theta_{k}=T \wedge \inf \left\{t \geq \hat{\sigma}_{k-1}: \mathbf{V}_{t}^{S_{\hat{\sigma}_{k-1}}^{(s)}, \hat{Z}_{k-1}, \hat{\gamma}_{k-1}}<0\right\}$ and the stochastic process $\left\{\mathbf{R}_{t}^{(k)}\right\}_{t=\hat{\sigma}_{k-1}}^{\Theta_{k}}$

$$
\begin{aligned}
& \mathbf{R}_{t}^{(k)}=\text { essinf } \inf _{\sigma \in \mathcal{T}_{T}, \hat{\sigma}_{k-1} \leq \sigma \leq \Theta_{k}} \text { ess } \sup _{\tau \in \mathcal{T}_{T}, \hat{\sigma}_{k-1} \leq \tau \leq \Theta_{k}} \\
& \mathbb{E}_{\mathbb{P}}\left(\mathbb{I}_{\tau} \leq \sigma\left(Y_{\tau}^{(s)}-\mathbf{V}_{\tau}^{S_{\tilde{\sigma}_{k-1}}^{(s)}, \hat{Z}_{k-1}, \hat{\gamma}_{k-1}}\right)^{+}+\right. \\
& \left.\mathbb{I}_{\sigma<\tau} \hat{R}\left(T-\sigma, S^{(s)}, \mathbf{V}_{\sigma}^{S_{\sigma_{k-1}}^{(s)}, \hat{z}_{k-1}, \hat{\gamma}_{k-1}}, \hat{\gamma}_{k-1}\right) \mid \mathcal{F}_{t}\right) .
\end{aligned}
$$

Next, introduce the random time

$$
\hat{\sigma}_{k}=\Theta_{k} \wedge \inf \left\{t \geq \hat{\sigma}_{k-1}: \mathbf{R}_{t}^{(k)}=\hat{R}\left(T-t, S^{(s)}, \mathbf{V}_{t}^{S_{\tilde{\sigma}_{k-1}}^{(s)}, \hat{z}_{k-1}, \hat{\gamma}_{k-1}}, \hat{\gamma}_{k-1}\right)\right\}
$$

and the random variable

$$
\hat{\beta}_{k}=\beta^{*}\left(T-\hat{\sigma}_{k}, S^{(s)}, \mathbf{V}_{\hat{\sigma}_{k}}^{S_{\hat{\sigma}_{k-1}}^{(s)}, \hat{z}_{k-1}, \hat{\gamma}_{k-1}}, \hat{\gamma}_{k-1}\right)
$$

where the function $\beta^{*}$ was introduced in Proposition 5.5. Finally, set

$$
\hat{\gamma}_{k}=\hat{\gamma}_{k-1}+\hat{\beta}_{k} \text { and } \hat{Z}_{k}=h\left(S_{\hat{\sigma}_{k}}^{(s)}, \mathbf{V}_{\tau}^{S_{\sigma_{k-1}}^{(s)}, \hat{Z}_{k-1}, \hat{\gamma}_{k-1}}, \hat{\gamma}_{k-1}, \hat{\beta}_{k}\right),
$$

with the function $h$ given by (3.1).

The following lemma completes the proof of Theorem 2.1 and gives a characterisation of the optimal hedge. 
Lemma 3.2. For any $k \geq 1$ the stochastic process $\left\{\mathbf{R}_{t}^{(k)}\right\}_{t=\hat{\sigma}_{k-1}}^{\Theta_{k}}$ is well defined, continuous and $\hat{\sigma}_{k}, \Theta_{k}$ are stopping times. Furthermore, the random variables $\hat{\gamma}_{k}, \hat{Z}_{k}$ are $\mathcal{F}_{\hat{\sigma}_{k}}$ measurable. Next, the structure of an optimal hedge is described in the following way. Set,

$$
\begin{gathered}
\hat{N}=\min \left\{k: \hat{\sigma}_{k}=T\right\} \wedge \\
\min \left\{k: \hat{R}\left(T-\hat{\sigma}_{k}, S^{(s)}, \mathbf{V}_{\hat{\sigma}_{k} k-1}^{S_{\hat{\sigma}_{k}}^{(s)}, \hat{z}_{k-1}, \hat{\gamma}_{k-1}}, \hat{\gamma}_{k-1}\right)=\left(X_{\hat{\sigma}_{k}}^{(s)}-\mathbf{V}_{\hat{\sigma}_{k}}^{S_{\hat{\sigma}_{k-1}}^{(s)}, \hat{z}_{k-1}, \hat{\gamma}_{k-1}}\right)^{+}\right\} .
\end{gathered}
$$

Then $\hat{N}<\infty$ a.s and the hedge $(\hat{\pi}, \hat{\sigma}) \in \mathcal{A}(T, s, z, y)$ which is given by $\hat{\pi}=(z, y, \hat{\gamma})$, where

$$
\hat{\gamma}_{t}=y \mathbb{I}_{t=0}+\sum_{i=1}^{\hat{N}-1} \hat{\gamma}_{i} \mathbb{I}_{\left(\hat{\sigma}_{i}, \hat{\sigma}_{i+1}\right]} \text { and } \hat{\sigma}=\hat{\sigma}_{\hat{N}},
$$

satisfies $R(T, s, z, y)=\mathcal{R}(T, s, \hat{\pi}, \hat{\sigma})$.

Proof. First, we establish a stronger version of the inequality (3.6). The exact form is the following. For any stopping time $\theta \in \mathcal{T}_{T}$ and random variables $Z \geq 0, Y$ which are $\mathcal{F}_{\theta}$ measurable,

$$
\begin{gathered}
R\left(T-\theta, S^{(s)}, Z, Y\right) \geq \inf _{\sigma \in \mathcal{T}_{T}, \theta \leq \sigma \leq \Theta} \sup _{\tau \in \mathcal{T}_{T}, \theta \leq \tau \leq \Theta} \\
\mathbb{E}_{\mathbb{P}}\left(\mathbb{I}_{\tau \leq \sigma}\left(Y_{\tau}^{(s)}-\mathbf{V}_{\tau}^{S_{\theta}^{(s)}, Z, Y}\right)^{+}+\mathbb{I}_{\sigma<\tau} \hat{R}\left(T-\sigma, S^{(s)}, \mathbf{V}_{\sigma}^{S_{\theta}^{(s)}, Z, Y}, Y\right)\right),
\end{gathered}
$$

where $\Theta=T \wedge \inf \left\{t \geq \theta: \mathbf{V}_{t}^{S_{\theta}^{(s)}, Z, Y}<0\right\}$. In order to derive (3.7) introduce the stochastic processes

$$
\begin{gathered}
\mathbf{X}_{t}:=\hat{R}\left(T-t, S^{(s)}, \mathbf{V}_{t}^{S_{\theta}^{(s)}, Z, Y}, Y\right), t \in[\theta, \Theta] \\
\mathbf{Y}_{t}:=\left(Y_{t}^{(s)}-\mathbf{V}_{t}^{S_{\theta}^{(s)}, Z, Y}\right)^{+}, t \in[\theta, \Theta] .
\end{gathered}
$$

From Proposition [5.5 it follows that the above processes are continuous. Observe that (since the buyer can stop at 0 ) for any $(\hat{\mathbf{T}}, \hat{v}, \hat{z}, \hat{y}) \in[0, T] \times C_{++}[0, T] \times \mathbb{R}_{+} \times \mathbb{R}$, $\hat{R}(\hat{\mathbf{T}}, \hat{v}, \hat{z}, \hat{y}) \geq\left(Y_{0}^{\hat{\mathbf{T}}, \hat{v}}-\hat{z}\right)^{+}$. We conclude that $\mathbf{X} \geq \mathbf{Y}$, and so by applying standard results on Dynkin games (see [18) we derive (3.7) from (3.6).

Next for a given $k$, consider the stochastic processes

$$
\begin{gathered}
\hat{\mathbf{X}}_{t}:=\hat{R}\left(T-t, S^{(s)}, \mathbf{V}_{t}^{S_{\sigma_{k-1}}^{(s)}, \hat{Z}_{k-1}, \hat{\gamma}_{k-1}},, \hat{\gamma}_{k-1}\right), t \in\left[\hat{\sigma}_{k-1}, \Theta_{k}\right], \\
\hat{\mathbf{Y}}_{t}=\left(Y_{t}^{(s)}-\mathbf{V}_{t}^{S_{\tilde{\sigma}_{k-1}}^{(s)}, \hat{z}_{k-1}, \hat{\gamma}_{k-1}}\right)^{+}, t \in\left[\hat{\sigma}_{k-1}, \Theta_{k}\right]
\end{gathered}
$$

where $\hat{Z}_{k}, \hat{\gamma}_{k}, \hat{\sigma}_{k}, \Theta_{k}, k \in \mathbb{N}$ were define above. Observe that $\hat{\mathbf{X}} \geq \hat{\mathbf{Y}}$. Thus, (induction on $k$ ) by applying Proposition 3.9 from [10. (see also Theorem 4.1 in [2]) we conclude that $\left\{\mathbf{R}_{t}^{(k)}\right\}_{t=\hat{\sigma}_{k-1}}^{\Theta_{k}}$ is a continuous stochastic process and $\hat{\sigma}_{k}$ is a stopping time, and $\hat{\gamma}_{k}, \hat{Z}_{k}$ are $\mathcal{F}_{\hat{\sigma}_{k}}$ measurable. From the definition of the function $\hat{R}$ it follows that $\hat{Z}_{k} \geq 0$ for $k \leq \hat{N}$. This together with the definition of the 
stopping times $\Theta_{k}, k \in \mathbb{N}$ and the fact that the portfolio value is constant after $\hat{\sigma}_{\hat{N}}$ yields that $\hat{\pi}$ is an admissible portfolio. Observe, that for any $n \in \mathbb{N}$ we have

$$
0 \leq V_{\hat{\sigma}_{n}}^{\hat{\pi}} \leq z+\max (\delta, \mu|y| s)+\sum_{i=1}^{n} \gamma_{\hat{\sigma}_{i-1}}^{\pi}\left(S_{\hat{\sigma}_{i}}^{(s)}-S_{\hat{\sigma}_{i-1}}^{(s)}\right)-n \delta \mathbb{I}_{\hat{N}>n}
$$

Taking the expectation with respect to the martingale measure $\mathbb{Q}$ we obtain that $\mathbb{Q}(\hat{N}>n) \leq \frac{z+\max (\delta, \mu|y| s)}{n}$, and so $\hat{N}<\infty$ a.s. Thus $(\hat{\pi}, \hat{\sigma}) \in \mathcal{A}(T, s, z, y)$.

Finally, we prove that $(\hat{\pi}, \hat{\sigma})$ is an optimal hedge. Choose $\epsilon>0$. There exists $N \in \mathbb{N}$ such that

$$
\mathbb{P}(\hat{N}>N)<\epsilon .
$$

Define the continuous martingale $M_{t}=\mathbb{E}_{\mathbb{P}}\left(\sup _{0 \leq t \leq T}\left(X_{t}^{(s)}\right)^{2} \mathbb{I}_{\hat{N}>N} \mid \mathcal{F}_{t}\right), t \in$ $[0, T]$, and the stochastic process

$$
\hat{U}_{t}=e s s \sup _{\tau \geq t, \tau \in \mathcal{T}_{T}} \mathbb{E}_{\mathbb{P}}\left(\left(\mathbb{I}_{\tau \leq \hat{\sigma}} Y_{\tau}^{(s)}+\mathbb{I}_{\hat{\sigma}<\tau} X_{\hat{\sigma}}^{(s)}-V_{\hat{\sigma} \wedge \tau}^{\hat{\pi}}\right)^{+} \mid \mathcal{F}_{t}\right), t \in[0, T] .
$$

Let us show that for any $0 \leq k \leq N$,

$$
\hat{U}_{\hat{\sigma}_{k \wedge \hat{N}}} \leq \hat{R}\left(T-\hat{\sigma}_{k \wedge \hat{N}}, S^{(s)}, V_{\hat{\sigma}_{k \wedge \hat{N}}^{\hat{\pi}}}, \hat{\gamma}_{k \wedge \hat{N}-1}\right)+\sqrt{M_{\hat{\sigma}_{k \wedge \hat{N}}}},
$$

where for $k=0$ we set $\hat{R}\left(T, S^{(s)}, z, \hat{\gamma}_{-1}\right):=R(T, s, z, y)$.

We start with $k=N$. From the definition of $\hat{N}$ and the Jensen inequality it follows that

$$
\begin{aligned}
& \hat{U}_{\hat{\sigma}_{N \wedge \hat{N}}} \leq \mathbb{I}_{\hat{\sigma}_{N \wedge \hat{N}}=\hat{\sigma}}\left(X_{\hat{\sigma}_{N \wedge \hat{N}}^{(s)}}-V_{\hat{\sigma}_{N \wedge \hat{N}}^{\hat{\pi}}}\right)^{+}+\mathbb{I}_{N<\hat{N}} \mathbb{E}_{\mathbb{P}}\left(\sup _{0 \leq t \leq \mathbb{T}}\left(X_{t}^{(s)}\right) \mid \mathcal{F}_{\hat{\sigma}_{N \wedge \hat{N}}}\right) \\
& \leq \hat{R}\left(T-\hat{\sigma}_{N \wedge \hat{N}}, S^{(s)}, V_{\hat{\sigma}_{N \wedge \hat{N}}^{\hat{\pi}}}, \hat{\gamma}_{N \wedge \hat{N}-1}\right)+\sqrt{M_{\hat{\sigma}_{N \wedge \hat{N}}}} \text {. }
\end{aligned}
$$

Next, we prove that if (3.9) is true for $k+1$ then it is true for $k$. From the definition of $\hat{N}$, on the event $k \geq \hat{N}$ (which is $\mathcal{F}_{\hat{\sigma}_{k \wedge \hat{N}}}$ measurable), (3.9) trivially holds true. Consider the event $k<\hat{N}$. On this event, similarly to (3.4) we have

$$
\hat{U}_{\hat{\sigma}_{k}}=\sup _{\tau \in \mathcal{T}_{T}} \mathbb{E}_{\mathbb{P}}\left(\mathbb{I}_{\hat{\sigma}_{k+1}<\tau} \hat{U}_{\hat{\sigma}_{k+1}}+\mathbb{I}_{\tau \leq \hat{\sigma}_{k+1}}\left(Y_{\tau}^{(s)}-\mathbf{V}_{\tau}^{S_{\sigma_{k}}^{(s)}, \hat{z}_{k}, \hat{\gamma}_{k}}\right)^{+} \mid \mathcal{F}_{\hat{\sigma}_{k \wedge \hat{N}}}\right) \leq
$$

from the induction assumption, since $\sqrt{M}$ is a super-martingale and by the definition of $\hat{\sigma}_{k+1}$,

$$
\begin{gathered}
\leq \sup _{\tau \in \mathcal{T}_{T}} \mathbb{E}_{\mathbb{P}}\left(\mathbb{I}_{\tau \leq \hat{\sigma}_{k+1}}\left(Y_{\tau}^{(s)}-\mathbf{V}_{\tau}^{S_{\hat{\sigma}_{k}}^{(s)}, \hat{Z}_{k}, \hat{\gamma}_{k}}\right)^{+}+\mathbb{I}_{\hat{\sigma}_{k+1}<\tau} \times\right. \\
\left.\hat{R}\left(T-\hat{\sigma}_{k+1}, S^{(s)}, V_{\hat{\sigma}_{k+1}}^{\hat{\pi}}, \hat{\gamma}_{k}\right) \mid \mathcal{F}_{\hat{\sigma}_{k \wedge \hat{N}}}\right)+\sqrt{M_{\hat{\sigma}_{k \wedge \hat{N}}}}= \\
\sqrt{M_{\hat{\sigma}_{k \wedge \hat{N}}}}+e s s \inf _{\sigma \in \mathcal{T}_{T}, \hat{\sigma}_{k} \leq \sigma \leq \Theta_{k+1}} \text { ess } \sup _{\tau \in \mathcal{T}_{T}, \hat{\sigma}_{k} \leq \tau \leq \Theta_{k+1}} \\
\mathbb{E}_{\mathbb{P}}\left(\mathbb{I}_{\tau \leq \sigma}\left(Y_{\tau}^{(s)}-\mathbf{V}_{\tau}^{S_{\sigma_{k}}^{(s)}, \hat{Z}_{k}, \hat{\gamma}_{k}}\right)^{+}+\mathbb{I}_{\sigma<\tau} \hat{R}\left(T-\sigma, S^{(s)}, \mathbf{V}_{\sigma}^{S_{\sigma_{k}}^{(s)}, \hat{Z}_{k}, \hat{\gamma}_{k}}, \hat{\gamma}_{k}\right) \mid \mathcal{F}_{\hat{\sigma}_{k \wedge \hat{N}}}\right) \leq
\end{gathered}
$$

by (3.7),

$\leq \sqrt{M_{\hat{\sigma}_{k \wedge \hat{N}}}}+R\left(T-\hat{\sigma}_{k}, S^{(s)}, V_{\hat{\sigma}_{k}}^{\hat{\pi}}, \hat{\gamma}_{k}\right)=\sqrt{M_{\hat{\sigma}_{k \wedge \hat{N}}}}+\hat{R}\left(T-\hat{\sigma}_{k \wedge \hat{N}}, S^{(s)}, V_{\hat{\sigma}_{k}}^{\hat{\pi}}, \hat{\gamma}_{k \wedge \hat{N}-1}\right)$ 
where the last equality follows from the definition of $\hat{\gamma}_{k}$ and the fact that we are on the event $k<\hat{N}$. This completes the proof of (3.9).

From (2.3), the Cauchy-Schwarz inequality and (3.8) it follows that $M_{0}=$ $O\left(\epsilon^{1 / 2}\right)$. Thus by applying (3.9) for $k=0$,

$$
\mathcal{R}(T, s, \hat{\pi}, \hat{\sigma})=\hat{U}_{0} \leq \sqrt{M_{0}}+R(T, s, z, y)=O\left(\epsilon^{1 / 4}\right)+R(T, s, z, y),
$$

and by taking $\epsilon \downarrow 0$ we complete the proof.

\section{Proof of Theorem 2.2}

First, we follow Lemma 4.1 in $[3$ and obtain a bound on the growth of admissible portfolios.

Lemma 4.1. Let $(z, y) \in \mathbb{R}_{++} \times \mathbb{R}$ be an initial position. There exists a constant $\tilde{C}$ such that for any $\pi \in \mathcal{A}(T, s, z, y)$,

$$
\mathbb{E}_{\mathbb{Q}}\left(\max _{0 \leq t \leq T}\left|\gamma_{t}\right| S_{t}^{(s)}+\int_{0}^{T} S_{u}^{(s)}\left|d \gamma_{u}\right|\right)^{2} \leq \tilde{C}\left(1+z^{2}+y^{2}\right) .
$$

Proof. Let $\pi=(z, y, \gamma) \in \mathcal{A}(T, s, z, y)$. We will use the integration by parts formula

$$
\int_{0}^{T} \gamma_{u} d S_{u}^{(s)}=\gamma_{t} S_{t}^{(s)}-y s-\int_{[0, t]} S_{u}^{(s)} d \gamma_{u}
$$

and the decomposition $\gamma_{t}=\gamma_{t}^{+}-\gamma_{t}^{-}$into a positive variation $\gamma^{+}$and a negative variation $\gamma^{-}$. From (2.4) and (4.1) it follows that for any $t \in[0, T]$

$0 \leq V_{t}^{\pi} \leq z+g(y, s)+|y| s-(1+\mu) \int_{[0, t]} S_{u}^{(s)} d \gamma_{u}-2 \mu \int_{[0, t]} S_{u}^{(s)} d \gamma_{u}^{-}+(1+\mu) \gamma_{t} S_{t}^{(s)}$

and

$0 \leq V_{t}^{\pi} \leq z+g(y, s)+|y| s-(1-\mu) \int_{[0, t]} S_{u}^{(s)} d \gamma_{u}-2 \mu \int_{[0, t]} S_{u}^{(s)} d \gamma_{u}^{+}+(1-\mu) \gamma_{t} S_{t}^{(s)}$.

This together with (4.1) yields

$$
\begin{gathered}
\int_{0}^{t} S_{u}^{(s)} d \gamma_{u}^{-} \leq \frac{1}{2 \mu}\left(z+g(y, s)+|y| s+(1+\mu)\left(|y| s+\int_{0}^{t} \gamma_{u} d S_{u}^{(s)}\right)\right) \\
\text { and } \int_{0}^{t} S_{u}^{(s)} d \gamma^{+} u \leq \frac{1}{2 \mu}\left(z+g(y, s)+|y| s+(1-\mu)\left(|y| s+\int_{0}^{t} \gamma_{u} d S_{u}^{(s)}\right)\right) .
\end{gathered}
$$

Consequently,

$$
\begin{gathered}
\gamma_{t} S_{t}^{(s)} \geq \int_{0}^{t} \gamma_{u} d S_{u}^{(s)}-\int_{[0, t]} S_{u}^{(s)} d \gamma_{u}^{-}-|y| s \geq \\
-\frac{1}{2 \mu}(z+g(y, s)+(2+3 \mu)|y| s)-\frac{1-\mu}{2 \mu} \int_{0}^{t} \gamma_{u} S_{u}^{(s)}
\end{gathered}
$$

and

$$
\begin{aligned}
& \gamma_{t} S_{t}^{(s)} \leq|y| s+\int_{0}^{t} \gamma_{u} d S_{u}^{(s)}+\int_{[0, t]} S_{u}^{(s)} d \gamma_{u}^{+} \leq \\
& \frac{1}{2 \mu}(z+g(y, s)+(2+\mu)|y| s)-\frac{1+\mu}{2 \mu} \int_{0}^{t} \gamma_{u} S_{u}^{(s)}
\end{aligned}
$$

From (4.3)-(4.4) and the inequality $(a+b)^{2} \leq 2\left(a^{2}+b^{2}\right)$ we obtain

$$
\left|\gamma_{t} S_{t}^{(s)}\right|^{2} \leq \frac{(z+g(y, s)+(2+3 \mu)|y| s)^{2}}{2 \mu^{2}}+\frac{(1+\mu)^{2}}{2 \mu^{2}}\left(\int_{0}^{t} \gamma(u) d S(u)\right)^{2} .
$$


Following the arguments of Lemma 4.1 in 3 we see that there is a constant $\tilde{c}$ such that

$$
\mathbb{E}_{\mathbb{Q}}\left(\sup _{0 \leq t \leq T}\left(\int_{0}^{t} \gamma_{u} d S_{u}^{(s)}\right)^{2}\right) \leq \tilde{c}(z+g(y, s)+(2+3 \mu)|y| s)^{2} .
$$

This together with (4.2) and (4.5) competes the proof.

Next, for any $n \in \mathbb{N}$ introduce the piecewise constant stochastic process $\hat{S}_{t}^{n, s}=$ $S_{\theta_{[n t / T]}^{(n)}}^{(s)}, t \in[0, T]$ and the payoffs

$$
\hat{Y}_{t}^{n, s}=\left[F\left(\hat{S}^{n, s}\right)\right]([n t / T] T / n) \leq \hat{X}_{t}^{n, s}=\left[G\left(\hat{S}^{n, s}\right)\right]([n t / T] T / n) \quad t \in[0, T] .
$$

By applying the results of Section 4 in [13] we get that there exists a constant $C_{1}$ such that for any $n$,

$$
\mathbb{E}_{\mathbb{P}}\left(\max _{1 \leq k \leq n} \max _{\theta_{k-1}^{(n)} \leq t \leq \theta_{k}^{(n)}}\left(\left|X_{t \wedge T}^{(s)}-\hat{X}_{\frac{k T}{n}}^{n, s}\right|+\left|Y_{t \wedge T}^{(s)}-\hat{Y}_{\frac{k T}{n}}^{n, s}\right|\right)\right) \leq C_{1} n^{-1 / 4}(\ln n)^{3 / 4} .
$$

Let $\hat{\mathcal{T}}_{n}$ the set of all stopping times with respect to the filtration $\left\{\mathcal{F}_{\theta_{k}^{(n)}}\right\}_{k=0}^{n}$ with values $k=0,1, \ldots, n$. Set,

$$
\hat{R}_{n}(T, s, z, y)=\inf _{\pi \in \mathcal{A} W, n} \inf _{(s, z, y)} \sup _{\zeta \in \hat{\mathcal{T}}_{n}} \mathbb{E}_{\eta \in \hat{\mathcal{T}}_{n}}\left(\hat{X}_{\frac{\zeta T}{n}}^{n, s} \mathbb{I}_{\zeta<\eta}+\hat{Y}_{\frac{\eta T}{n}}^{n, s} \mathbb{I}_{\eta \leq \zeta}-V_{\theta_{\zeta \wedge \eta}^{(n)}}^{\pi}\right)^{+}
$$

Observe that $\hat{S}_{t}^{n, s}=\Pi_{n}\left(S_{t}^{n, s}\right), \hat{Y}_{t}^{n, s}=\Pi_{n}\left(Y_{t}^{n, s}\right)$ and $\hat{X}_{t}^{n, s}=\Pi_{n}\left(X_{t}^{n, s}\right), t \in[0, T]$. Thus by using similar arguments as in [3] and [6] (see Section 3 in these papers) we get that

$$
\hat{R}_{n}(T, s, z, y)=R_{n}(T, s, z, y) .
$$

The equality (4.7) plays a key role in the proof of the following result.

Lemma 4.2. Let $(z, y) \in \mathbb{R}_{++} \times \mathbb{R}$ be an initial position. There exists a constant $C_{2}>0$ such that for any $n \in \mathbb{N}$

$$
R(T, s, z, y) \geq R_{n}(T, s, z, y)-C_{2} n^{-1 / 4}(\ln n)^{3 / 4} .
$$

Proof. Fix $n$ and set $z_{n}=z-n^{-1 / 3}$. Assume that $n$ is sufficiently large, so $z_{n} \geq 0$. Let $(\pi, \sigma) \in \mathcal{A}\left(T, s, z_{n}, y\right)$ be such that

$$
R\left(T, s, z_{n}, y\right)>\mathcal{R}(T, s, \pi, \sigma)-\frac{1}{n} .
$$

Introduce the random variable

$$
\Gamma=\max _{0 \leq t \leq T}\left|\gamma_{t}\right| S_{t}^{(s)}+\int_{0}^{T} S_{u}^{(s)}\left|d \gamma_{u}\right|
$$

Define the stopping time

$$
\Upsilon=T \wedge \inf \left\{t: \max _{0 \leq u \leq t}\left|\gamma_{u}\right| S_{u}^{(s)}+\int_{0}^{t} S_{u}^{(s)}\left|d \gamma_{u}\right|>n^{1 / 7}\right\}
$$

and the portfolio $\dot{\pi}=(z, y, \dot{\gamma})$ by $\dot{\gamma}_{t}=\gamma_{t} \mathbb{I}_{t \leq \Upsilon}$. Namely, we liquidate the portfolio at the stopping time $\Upsilon$. Observe that the initial capitals of the portfolios $\dot{\pi}$ and $\pi$, equal to $z$ and $z_{n}$, respectively. From Lemma 4.1 and the Chebyshev inequality it follows that

$$
\mathbb{Q}(\Upsilon<T)=\mathbb{Q}\left(\Gamma>n^{1 / 7}\right)=O\left(n^{-2 / 7}\right) .
$$


This together with (2.2)-(2.3) and the Hölder inequality (for $p=8, q=8 / 7$ ) gives

$$
\mathcal{R}(T, s, \dot{\pi}, \sigma)-\mathcal{R}(T, s, \pi, \sigma) \leq \mathbb{E}_{\mathbb{Q}}\left(Z_{T}^{-1} \sup _{0 \leq t \leq T} X_{t}^{(s)} \mathbb{I}_{\Upsilon<T}\right)=O\left(n^{-1 / 4}\right) .
$$

Introduce the portfolio $\tilde{\pi}=(z, y, \tilde{\gamma}) \in \mathcal{A}^{W, n}(s, z, y)$ which is managed at the stopping times $0, \theta_{1}^{(n)}, \ldots, \theta_{n}^{(n)}$ and is given by

$$
\tilde{\gamma}_{t}=y \mathbb{I}_{t=0}+\sum_{i=0}^{n-1} \dot{\gamma}_{\theta_{i}^{(n)}} \mathbb{I}_{\left(\theta_{i}^{(n)}, \theta_{i+1}^{(n)}\right]} .
$$

Let $t \in[0, T]$ and consider the event $t \in\left(\theta_{k}^{(n)}, \theta_{k+1}^{(n)}\right]$ for some $k<n$. From the integration by parts formula we get

$$
\begin{aligned}
& V_{t}^{\dot{\pi}}=z_{n}+\dot{\gamma}_{t} S_{t}^{(s)}-y s-\left(\sum_{i=1}^{k} \int_{\left[\theta_{i-1}^{(n)}, \theta_{i}^{(n)}\right)} S_{u}^{(s)} d \dot{\gamma}_{u}-\int_{\left[\theta_{k}^{(n)}, t\right)} S_{u}^{(s)} d \dot{\gamma}_{u}\right)- \\
& \left(\sum_{i=1}^{k} \sum_{u \in\left[\theta_{i-1}^{(n)}, \theta_{i}^{(n)}\right)} g\left(\dot{\gamma}_{u+}-\dot{\gamma}_{u}, S_{u}^{(s)}\right)+\sum_{u \in\left[\theta_{k}^{(n)}, t\right)} g\left(\dot{\gamma}_{u+}-\dot{\gamma}_{u}, S_{u}^{(s)}\right)\right) \leq
\end{aligned}
$$

since $\left|S_{u}^{(s)}-S_{\theta_{k+1}^{(n)}}^{(s)}\right| \leq 4 \kappa \sqrt{\frac{T}{n}} \min \left(S_{u}^{(s)}, S_{\theta_{k+1}^{(n)}}^{(s)}\right)$ for $u \in\left[\theta_{k}^{(n)}, \theta_{k+1}^{(n)}\right]$ and large $n$

$$
\begin{gathered}
\leq z_{n}+\dot{\gamma}_{t} S_{\theta_{k+1}^{(n)}}^{(s)}+4 \kappa \dot{\gamma}_{t} \sqrt{\frac{T}{n}} S_{t}^{(s)}-y s- \\
\sum_{i=1}^{k+1} S_{\theta_{i}^{(n)}}^{(s)} \int_{\left[\theta_{i-1}^{(n)}, \theta_{i}^{(n)}\right)} d \dot{\gamma}_{u}+4 \kappa \sqrt{\frac{T}{n}} \int_{0}^{T} S_{u}^{(s)}\left|d \dot{\gamma}_{u}\right|- \\
\sum_{i=1}^{k} g\left(\dot{\gamma}_{\theta_{i}^{(n)}}-\dot{\gamma}_{\theta_{i-1}^{(n)}}, S_{\theta_{i}^{(n)}}^{(s)}\right)+4 \kappa \mu \sqrt{\frac{T}{n}} \int_{0}^{T} S_{u}^{(s)}\left|d \dot{\gamma}_{u}\right|-\int_{\left[\theta_{k}^{(n)}, t\right)} \mu S_{t}^{(s)}\left|d \dot{\gamma}_{t}\right| \leq
\end{gathered}
$$

since $\max _{0 \leq u \leq T}\left|\dot{\gamma}_{u}\right| S_{u}^{(s)}+\int_{0}^{T} S_{u}^{(s)}\left|d \dot{\gamma}_{u}\right| \leq n^{1 / 7}$, then for large $n$

$$
\begin{gathered}
\leq z-y s+\dot{\gamma}_{\theta_{k}^{(n)}} S_{\theta_{k+1}^{(n)}}^{(s)}+S_{\theta_{k+1}^{(n)}}^{(s)} \int_{\left[\theta_{k}^{(n)}, t\right)}\left|d \dot{\gamma}_{u}\right|-\sum_{i=1}^{k+1} S_{\theta_{i}^{(n)}}^{(s)} \int_{\left[\theta_{i-1}^{(n)}, \theta_{i}^{(n)}\right)} d \dot{\gamma}_{u} \\
-\sum_{i=1}^{k} g\left(\dot{\gamma}_{\theta_{i}^{(n)}}-\dot{\gamma}_{\theta_{i-1}^{(n)}}, S_{\theta_{i}^{(n)}}^{(s)}\right)-\int_{\left[\theta_{k}^{(n)}, t\right)} \mu S_{t}^{(s)}\left|d \dot{\gamma}_{t}\right| \leq \\
z-y s+\dot{\gamma}_{\theta_{k}^{(n)}} S_{\theta_{k+1}^{(n)}}^{(s)}-\sum_{i=1}^{k+1} S_{\theta_{i+1}^{(s)}}^{(s)} \int_{\left[\theta_{i-1}^{(n)}, \theta_{i}^{(n)}\right)} d \dot{\gamma}_{u} \\
-\sum_{i=1}^{k} g\left(\dot{\gamma}_{\theta_{i}^{(n)}}-\dot{\gamma}_{\theta_{i-1}^{(n)}}, S_{\theta_{i}^{(n)}}^{(s)}\right)=V_{\theta_{k+1}^{(n)}}^{\tilde{\pi}}
\end{gathered}
$$

where the last equality follows from the summation by parts. We conclude that (for sufficiently large $n$ )

$$
V_{\theta_{k+1}^{(n)}}^{\tilde{\pi}} \geq V_{t}^{\dot{\pi}}, \quad t \in\left(\theta_{k}^{(n)}, \theta_{k+1}^{(n)}\right], \quad k<n .
$$

In particular, $\tilde{\pi} \in \mathcal{A}^{W, n}(s, z, y)$ is an admissible portfolio.

Let $\zeta \in \hat{\mathcal{T}}_{n}$ be given by

$$
\zeta=n \wedge \min \left\{k: \theta_{k}^{(n)} \geq \sigma\right\} \text { if } \sigma<T \text { and } \zeta=n \text { if } \sigma=T
$$


where $(\pi, \sigma)$ satisfies (4.8). From (4.7)-(4.9) and Lemma 5.3 we get

$$
\begin{gathered}
R_{n}(T, s, z, y)-R(T, s, z, y) \leq O\left(\left|z_{n}-z\right|^{3 / 4}\right)+\frac{1}{n}+O\left(n^{-1 / 4}\right)+ \\
\hat{R}_{n}(T, s, z, y)-R(T, s, \dot{\pi}, \sigma) \leq O\left(n^{-1 / 4}\right)+ \\
\sup _{\eta \in \hat{\mathcal{T}}_{n}} \mathbb{E}_{\mathbb{P}}\left(\hat{X}_{\frac{\zeta T}{n}}^{n, s} \mathbb{I}_{\zeta<\eta}+\hat{Y}_{\frac{\eta T}{n}}^{n, s} \mathbb{I}_{\eta \leq \zeta}-V_{\theta_{\zeta \wedge \eta}^{(n)}}^{\tilde{\pi}}\right)^{+}- \\
\sup _{\tau \in \mathcal{T}_{T}} \mathbb{E}_{\mathbb{P}}\left(X_{\sigma}^{(s)} \mathbb{I}_{\sigma<\tau}+Y_{\tau}^{(s)} \mathbb{I}_{\tau \leq \sigma}-V_{\sigma \wedge \tau}^{\dot{\pi}}\right)^{+} \leq
\end{gathered}
$$

since $T \wedge \theta_{\eta}^{(n)} \in \mathcal{T}_{T}$ for any $\eta \in \mathcal{T}_{n}$

$$
\begin{aligned}
& \leq O\left(n^{-1 / 4}\right)+\sup _{\eta \in \hat{\mathcal{T}}_{n}} \mathbb{E}_{\mathbb{P}}\left(\hat{X}_{\frac{\zeta T}{n}}^{n, s} \mathbb{I}_{\zeta<\eta}+\hat{Y}_{\frac{\eta T}{n}}^{n, s} \mathbb{I}_{\eta \leq \zeta}-V_{\theta_{\zeta \wedge \eta}^{(n)}}^{\tilde{\pi}}\right)^{+}- \\
& \sup _{\eta \in \hat{\mathcal{T}}_{n}} \mathbb{E}_{\mathbb{P}}\left(X_{\sigma}^{(s)} \mathbb{I}_{\sigma<\theta_{\eta}^{(n)} \wedge T}+Y_{\theta_{\eta}^{(n)} \wedge T}^{(s)} \mathbb{I}_{\theta_{\eta}^{(n)} \wedge T \leq \sigma}-V_{\sigma \wedge \theta_{\eta}^{(n)}}^{\dot{\pi}}\right)^{+} \leq
\end{aligned}
$$

since $\sigma<\theta_{\eta}^{(n)} \wedge T$ by (4.11) if $\zeta<\eta$

$$
\begin{gathered}
\leq O\left(n^{-1 / 4}\right)+\mathbb{E}_{\mathbb{P}}\left(\left|\hat{X}_{\frac{\zeta T}{n}}^{n, s}-X_{\sigma}^{(s)}\right| \mathbb{I}_{\sigma<\theta_{n}^{(n)}}\right)+\sup _{\eta \in \hat{\mathcal{T}}_{n}} \mathbb{E}_{\mathbb{P}}\left|\hat{Y}_{\frac{\eta_{n} T}{n}}^{n, s}-Y_{\theta_{\eta}^{(n)} \wedge T}^{(s)}\right|+ \\
\sup _{\eta \in \hat{\mathcal{T}}_{n}} \mathbb{E}_{\mathbb{P}}\left(V_{\sigma \wedge \theta_{\eta}^{(n)}}^{\dot{\pi}}-V_{\theta_{\zeta \wedge \eta}^{(n)}}^{\tilde{\pi}}\right)^{+} \leq
\end{gathered}
$$

since $V_{\theta_{\zeta \wedge \eta}^{(n)}}^{\dot{\pi}_{n}} \geq V_{\sigma \wedge \theta_{\eta}^{(n)}}^{\dot{\pi}}$ by (4.10), together with (4.6),

$$
\begin{gathered}
\leq O\left(n^{-1 / 4}\right)+\mathbb{E}_{\mathbb{P}}\left(\max _{1 \leq k \leq n} \max _{\theta_{k-1}^{(n)} \leq t \leq \theta_{k}^{(n)}}\left(\left|X_{t \wedge T}^{(s)}-\hat{X}_{\frac{k T}{n}}^{n, s}\right|+\left|Y_{t \wedge T}^{(s)}-\hat{Y}_{\frac{k T}{n}}^{n, s}\right|\right)\right) \\
=O\left(n^{-1 / 4}(\ln n)^{3 / 4}\right),
\end{gathered}
$$

and the result follows.

In view of Lemma 4.2, in order to complete the proof of Theorem 2.2 it remains to establish the following result.

Lemma 4.3. There exists a constant $C_{3}$ such that for any $n \in \mathbb{N}$ and $(\pi, \sigma) \in$ $\mathcal{A}^{(n)}(T, s, z, y)$,

$$
\mathcal{R}(T, s, \tilde{\pi}, \tilde{\sigma}) \leq \mathcal{R}_{n}(T, s, \pi, \sigma)+C_{3} n^{-1 / 4}(\ln n)^{3 / 4},
$$

for $(\tilde{\pi}, \tilde{\sigma})=\Psi_{n}(\pi, \sigma)$.

Proof. The proof follows the proof of (2.26) in [6]. In the proof of (2.26) in [6] we showed that if we lift a hedge to the BS model, the shortfall risk can increase only by the amount $O\left(n^{-1 / 4}(\ln n)^{3 / 4}\right)$. Though in 6 there is no friction, the only property that we used there is that the portfolio value process is a super-martingale with respect to the martingale measure $\mathbb{Q}$. In the current setup, this fact remains true, and so we just follow the proof from [6].

\section{Regularity PROPERTIES OF SHORTFAll RiSK}

In this section we do not assume Lipschitz continuity of the functions $F, G$ (that is (2.5)) but just continuity and (2.3). We start with the following lemma. 
Lemma 5.1. Let $v \in C_{++}[0, T]$. There exists a continuous function $m_{v}: \mathbb{R}_{+} \rightarrow \mathbb{R}_{+}$ (modulus of continuity) with $m_{v}(0)=0$ such that for any $\mathbf{T}_{1}, \mathbf{T}_{2} \in[0, T], z \geq 0$ and $y \in \mathbb{R}$, we have

$$
\left|R\left(\mathbf{T}_{1}, v, z, y\right)-R\left(\mathbf{T}_{2}, v, z, \frac{y v\left(T-\mathbf{T}_{1}\right)}{v\left(T-\mathbf{T}_{2}\right)}\right)\right| \leq m_{v}\left(\left|\mathbf{T}_{1}-\mathbf{T}_{2}\right|\right) .
$$

Proof. Let $\mathbf{T}_{1}, \mathbf{T}_{2} \in[0, T], z \geq 0$ and $y \in \mathbb{R}$. Without loss of generality, we assume that $\mathbf{T}_{1}<\mathbf{T}_{2}$. Choose $\epsilon>0$. There exists a hedge $\left(\pi_{1}, \sigma_{1}\right) \in \mathcal{A}\left(\mathbf{T}_{1}, v\left(T-\mathbf{T}_{1}\right), z, y\right)$ such that

$$
R\left(\mathbf{T}_{1}, v, z, y\right)>\mathcal{R}\left(\mathbf{T}_{1}, v, \pi_{1}, \sigma_{1}\right)-\epsilon .
$$

Set $\pi_{1}=\left(z, y, \gamma^{(1)}\right)$. Let $\left(\pi_{2}, \sigma_{2}\right) \in \mathcal{A}\left(\mathbf{T}_{2}, v\left(T-\mathbf{T}_{2}\right), z, \frac{y v\left(T-\mathbf{T}_{1}\right)}{v\left(T-\mathbf{T}_{2}\right)}\right)$ be a hedge such that $\pi_{2}=\left(z, y, \gamma^{(2)}\right)$ is given by $\gamma_{t}^{(2)}=\mathbb{I}_{t \leq \mathbf{T}_{1}} \frac{\gamma_{t}^{(1)} v\left(T-\mathbf{T}_{1}\right)}{v\left(T-\mathbf{T}_{2}\right)}, \quad t \in\left[0, \mathbf{T}_{2}\right]$, and $\sigma_{2}=\sigma_{1} \mathbb{I}_{\sigma_{1}<\mathbf{T}_{1}}+\mathbf{T}_{2} \mathbf{I}_{\sigma_{1}=\mathbf{T}_{1}}$. Namely, the portfolio $\gamma^{(2)}$ is proportional $\gamma^{(1)}$ until the moment $\mathbf{T}_{1}$ and then we sell the stocks. The stopping time $\sigma_{2}$ is almost the same as $\sigma_{1}$ with a small modification such that if $\sigma_{1}$ is equal to $\mathbf{T}_{1}$ then $\sigma_{2}=\mathbf{T}_{2}$. From (2.4) it follows that $\left(\pi_{2}, \sigma_{2}\right) \in \mathcal{A}\left(\mathbf{T}_{2}, v\left(T-\mathbf{T}_{2}\right), z, \frac{y v\left(T-\mathbf{T}_{1}\right)}{v\left(T-\mathbf{T}_{2}\right)}\right)$ and $V_{\sigma_{2} \wedge t}^{\pi_{2}}=V_{\sigma_{1} \wedge t}^{\pi_{1}}$, $t \in\left[0, \mathbf{T}_{2}\right]$.

Let $\tau \in \mathcal{T}_{\mathbf{T}_{2}}$. Observe that if $\sigma_{2}<\tau$ then $\sigma_{1}<\tau \wedge \mathbf{T}_{1}$. Thus from (5.1),

$$
\begin{gathered}
R\left(\mathbf{T}_{2}, v, z, \frac{y v\left(T-\mathbf{T}_{1}\right)}{v\left(T-\mathbf{T}_{2}\right)}\right) \leq \mathcal{R}\left(\mathbf{T}_{2}, v, \pi_{2}, \sigma_{2}\right)= \\
\sup _{\tau \in \mathcal{T}_{\mathbf{T}_{2}}} \mathbb{E}_{\mathbb{P}}\left(X_{\sigma_{2}}^{\mathbf{T}_{2}, v} \mathbb{I}_{\sigma_{2}<\tau}+Y_{\tau}^{\mathbf{T}_{2}, v} \mathbb{I}_{\tau \leq \sigma_{2}}-V_{\sigma_{2} \wedge \tau}^{\pi_{2}}\right)^{+} \leq \\
\sup _{\tau \in \mathcal{T}_{\mathbf{T}_{2}}} \mathbb{E}_{\mathbb{P}}\left(X_{\sigma_{1}}^{\mathbf{T}_{1}, v} \mathbb{I}_{\sigma_{1}<\tau \wedge \mathbf{T}_{1}}+Y_{\tau \wedge}^{\mathbf{T}_{1}, v} \mathbb{\mathbf { T }}_{1} \mathbb{I}_{\tau \wedge \mathbf{T}_{1} \leq \sigma_{1}}-V_{\sigma_{1} \wedge \tau}^{\pi_{1}}\right)^{+}+ \\
\mathbb{E}_{\mathbb{P}}\left(\sup _{0 \leq t \leq \mathbf{T}_{2}}\left(\left|Y_{t \wedge \mathbf{T}_{1}}^{\mathbf{T}_{1}, v}-Y_{t}^{\mathbf{T}_{2}, v}\right|+\left|X_{t \wedge \mathbf{T}_{1}}^{\mathbf{T}_{1}, v}-X_{t}^{\mathbf{T}_{2}, v}\right|\right)\right) \leq \\
\epsilon+R\left(\mathbf{T}_{1}, v, z, y\right)+m_{v}\left(\left|\mathbf{T}_{1}-\mathbf{T}_{2}\right|\right),
\end{gathered}
$$

where

$$
m_{v}(\delta)=\sup _{\left|t_{2}-t_{1}\right| \leq \delta} \mathbb{E}_{\mathbb{P}}\left(\sup _{0 \leq t \leq T}\left(\left|Y_{t \wedge t_{1}}^{t_{1}, v}-Y_{t}^{t_{2}, v}\right|+\left|X_{t \wedge t_{1}}^{t_{1}, v}-X_{t}^{t_{2}, v}\right|\right)\right) .
$$

From (2.3) and the fact that $F, G$ are continuous it follows that $m_{v}$ is indeed a modulus of continuity. Since $\epsilon>0$ was arbitrary we get

$$
R\left(\mathbf{T}_{2}, v, z, \frac{y v\left(T-\mathbf{T}_{1}\right)}{v\left(T-\mathbf{T}_{2}\right)}\right)-R\left(\mathbf{T}_{1}, v, z, y\right) \leq m_{v}\left(\left|\mathbf{T}_{1}-\mathbf{T}_{2}\right|\right) .
$$

Next, we prove

$$
R\left(\mathbf{T}_{1}, v, z, y\right)-R\left(\mathbf{T}_{2}, v, z, \frac{y v\left(T-\mathbf{T}_{1}\right)}{v\left(T-\mathbf{T}_{2}\right)}\right) \leq m_{v}\left(\left|\mathbf{T}_{1}-\mathbf{T}_{2}\right|\right) .
$$

Choose $\epsilon>0$ and a hedge $\left(\tilde{\pi}_{2}, \tilde{\sigma}_{2}\right) \in \mathcal{A}\left(\mathbf{T}_{2}, v\left(T-\mathbf{T}_{2}\right), z, \frac{y v\left(T-\mathbf{T}_{1}\right)}{v\left(T-\mathbf{T}_{2}\right)}\right)$ which satisfy

$$
R\left(\mathbf{T}_{2}, v, z, \frac{y v\left(T-\mathbf{T}_{1}\right)}{v\left(T-\mathbf{T}_{2}\right)}\right)>\mathcal{R}\left(\mathbf{T}_{2}, v, \tilde{\pi}_{2}, \tilde{\sigma}_{2}\right)-\epsilon .
$$

Denote $\tilde{\pi}_{2}=\left(z, \frac{y v\left(T-\mathbf{T}_{1}\right)}{v\left(T-\mathbf{T}_{2}\right)}, \tilde{\gamma}^{(2)}\right)$. Let $\left(\tilde{\pi}_{1}, \tilde{\sigma}_{1}\right) \in \mathcal{A}\left(\mathbf{T}_{1}, v\left(T-\mathbf{T}_{1}\right), z, y\right)$ be a hedge such that $\tilde{\pi}_{1}=\left(z, y, \tilde{\gamma}^{(1)}\right)$ is given by $\tilde{\gamma}_{t}^{(1)}=\frac{\gamma_{t}^{(2)} v\left(T-\mathbf{T}_{2}\right)}{v\left(T-\mathbf{T}_{1}\right)}, t \in\left[0, \mathbf{T}_{1}\right]$, and $\tilde{\sigma}_{1}=$ 
$\tilde{\sigma}_{2} \wedge \mathbf{T}_{1}$. Namely, we take a multiple of the hedge $\left(\tilde{\pi}_{2}, \tilde{\sigma}_{2}\right)$ and restrict it to the interval $\left[0, \mathbf{T}_{1}\right]$. Let $\tau \in \mathcal{T}_{\mathbf{T}_{1}}$ and observe that if $\sigma_{2}<\tau$ then $\sigma_{1}=\sigma_{2}<\tau$. Thus we obtain,

$$
\begin{gathered}
R\left(\mathbf{T}_{1}, v, z, y\right) \leq \mathcal{R}\left(\mathbf{T}_{1}, v, \tilde{\pi}_{1}, \tilde{\sigma}_{1}\right)= \\
\sup _{\tau \in \mathcal{T}_{\mathbf{T}_{1}}} \mathbb{E}_{\mathbb{P}}\left(X_{\tilde{\sigma}_{1}}^{\mathbf{T}_{1}, v} \mathbb{I}_{\tilde{\sigma}_{1}<\tau}+Y_{\tau}^{\mathbf{T}_{1}, v} \mathbb{I}_{\tau \leq \tilde{\sigma}_{1}}-V_{\tilde{\sigma}_{1} \wedge \tau}^{\tilde{\pi}_{1}}\right)^{+} \\
\leq \sup _{\tau \in \mathcal{T}_{\mathbf{T}_{1}}} \mathbb{E}_{\mathbb{P}}\left(X_{\tilde{\sigma}_{2}}^{\mathbf{T}_{2}, v} \mathbb{I}_{\tilde{\sigma}_{2}<\tau}+Y_{\tau}^{\mathbf{T}_{2}, v} \mathbb{I}_{\tau \leq \tilde{\sigma}_{2}}-V_{\tilde{\sigma}_{2} \wedge \tau}^{\tilde{\pi}_{2}}\right)^{+}+ \\
\mathbb{E}_{\mathbb{P}}\left(\sup _{0 \leq t \leq \mathbf{T}_{2}}\left(\left|Y_{t \wedge \mathbf{T}_{1}}^{\mathbf{T}_{1}, v}-Y_{t}^{\mathbf{T}_{2}, v}\right|+\left|X_{t \wedge \mathbf{T}_{1}}^{\mathbf{T}_{1}, v}-X_{t}^{\mathbf{T}_{2}, v}\right|\right)\right) \leq \\
\epsilon+R\left(\mathbf{T}_{2}, v, z, \frac{y v\left(T-\mathbf{T}_{1}\right)}{v\left(T-\mathbf{T}_{2}\right)}\right)+m_{v}\left(\left|\mathbf{T}_{1}-\mathbf{T}_{2}\right|\right),
\end{gathered}
$$

and by taking $\epsilon \downarrow 0$ we complete the proof.

Next, we obtain the following simple result.

Lemma 5.2. Let $v \in C_{++}[0, T]$. There exists a continuous function $\tilde{m}_{v}: \mathbb{R}_{+} \rightarrow \mathbb{R}_{+}$ with $\tilde{m}_{v}(0)=0$ such that for any $\tilde{v} \in C_{++}[0, T], \mathbf{T} \in[0, T], z \geq 0$ and $y \in \mathbb{R}$, we have

$$
\left|R(\mathbf{T}, v, z, y)-R\left(\mathbf{T}, \tilde{v}, z, \frac{y v(T-\mathbf{T})}{\tilde{v}(T-\mathbf{T})}\right)\right| \leq \tilde{m}_{v}(\|v-\tilde{v}\|) .
$$

Proof. Fix $\mathbf{T} \in[0, T], \tilde{v} \in C_{++}[0, T], z \geq 0$ and $y \in \mathbb{R}$. Let $\pi_{1}=(z, y, \gamma)$ be an admissible portfolio in the market with maturity date $\mathbf{T}$ and an initial stock price $v(T-\mathbf{T})$. Consider the portfolio $\pi_{2}=\left(z, \frac{y v(T-\mathbf{T})}{\tilde{v}(T-\mathbf{T})}, \frac{\gamma v(T-\mathbf{T})}{\tilde{v}(T-\mathbf{T})}\right)$ as an admissible portfolio in the market with maturity date $\mathbf{T}$ and an initial stock price $\tilde{v}(T-\mathbf{T})$. The map $\pi_{1} \rightarrow \pi_{2}$ is a bijection between the corresponding sets of portfolios. From (2.4) it follows that $V^{\pi_{1}}=V^{\pi_{2}}$, and so

$$
\begin{gathered}
\left|R(\mathbf{T}, v, z, y)-R\left(\mathbf{T}, \tilde{v}, z, \frac{y v(T-\mathbf{T})}{\tilde{v}(T-\mathbf{T})}\right)\right| \leq \\
\mathbb{E}_{\mathbb{P}}\left(\sup _{0 \leq t \leq \mathbf{T}}\left(\left|Y_{t}^{\mathbf{T}, v}-Y_{t}^{\mathbf{T}, \tilde{v}}\right|+\left|X_{t}^{\mathbf{T}, v}-X_{t}^{\mathbf{T}, \tilde{v}}\right|\right)\right) \leq \tilde{m}_{v}(\| v-\tilde{v}||)
\end{gathered}
$$

where

$$
\tilde{m}_{v}(\delta)=\sup _{\mathbf{T} \in[0, T]} \sup _{\|\tilde{v}-v\| \leq \delta} \mathbb{E}_{\mathbb{P}}\left(\left\|X^{\mathbf{T}, v}-X^{\mathbf{T}, \tilde{v}}\right\|+\left\|Y^{\mathbf{T}, v}-Y^{\mathbf{T}, \tilde{v}}\right\|\right) .
$$

From (2.3) and the fact that $F, G$ are continuous it follows that $\tilde{m}_{v}$ is indeed a modulus of continuity.

Next, we establish continuity properties of the shortfall risk as a function of the initial position.

Lemma 5.3. Let $K>0$. There exists a constant $\hat{C}=\hat{C}(K)$ such that the following holds. For any $v \in C_{++}[0, T], \mathbf{T} \in[0, T],\left(z_{i}, y_{i}\right) \in \mathbb{R}_{+} \times \mathbb{R}, i=1,2$, such that $\|v\| \leq K$ and $y_{1}=0 \Leftrightarrow y_{2}=0$, we have

$$
\left|R\left(\mathbf{T}, v, z_{1}, y_{1}\right)-R\left(\mathbf{T}, v, z_{2}, y_{2}\right)\right| \leq \hat{C}\left(\left|z_{1}-z_{2}\right|+\left|y_{1}-y_{2}\right|\right)^{3 / 4} .
$$

Proof. Fix $v \in C_{++}[0, T], \mathbf{T} \in[0, T]$ and $\left(z_{i}, y_{i}\right) \in \mathbb{R}_{+} \times \mathbb{R}, i=1,2$. Assume that $\|v\| \leq K$ and $y_{1}=0 \Leftrightarrow y_{2}=0$. Denote $s=v(T-\mathbf{T})$ and $A=\left|z_{1}-z_{2}\right|+\left|y_{1}-y_{2}\right|$. 
Without loss of generality we assume that $0<A<1$. Let $\left(\pi_{1}, \sigma_{1}\right) \in \mathcal{A}\left(\mathbf{T}, s, z_{1}, y_{1}\right)$ be such that

$$
\mathcal{R}\left(\mathbf{T}, v, \pi_{1}, \sigma_{1}\right)<R\left(\mathbf{T}, v, z_{1}, y_{1}\right)+A .
$$

Denote $\pi_{1}=\left(z, y, \gamma^{(1)}\right)$. Define the stopping times $\varrho=\inf \left\{t: \gamma_{t+}^{(1)}=0\right\} \wedge \mathbf{T}$ and

$$
\varsigma=\inf \left\{t: V_{t}^{\pi_{1}} \leq\left|z_{2}-z_{1}\right|+\left|y_{2}-y_{1}\right|(1+\mu)\left(s+S_{t \wedge \varrho}^{(s)}\right\} \wedge \mathbf{T} .\right.
$$

Introduce the hedge $\left(\pi_{2}, \sigma_{2}\right) \in \mathcal{A}\left(\mathbf{T}, s, z_{2}, y_{2}\right)$ by the relation $\sigma_{2}=\sigma_{1}$ and

$$
\gamma_{t}^{(2)}=\mathbb{I}_{t \leq \varsigma}\left(\left(\gamma_{t}^{(1)}+y_{2}-y_{1}\right) \mathbb{I}_{t \leq \varrho}+\gamma_{t}^{(1)} \mathbb{I}_{t>\varrho}\right) .
$$

Namely, at the time $\varsigma$ the investor liquidates the portfolio. Until the time $\varsigma$, the portfolio strategy $\gamma^{(2)}$ is a shift of $\gamma^{(1)}$ until the first stock liquidation time $\varrho$ of $\gamma^{(1)}$ and after this time the portfolios are the same. From (2.4) it follows that

$$
V_{t}^{\pi_{2}}=V_{t \wedge \varsigma}^{\pi_{2}} \geq V_{t \wedge \varsigma}^{\pi_{1}}-\left(\left|z_{2}-z_{1}\right|+\left|y_{2}-y_{1}\right|(1+\mu)\left(s+S_{t \wedge \varsigma \wedge \varrho}^{(s)}\right)\right) \geq 0
$$

where the last inequality follows from the definition of $\varsigma$. Thus $\left(\pi_{2}, \sigma_{2}\right) \in \mathcal{A}\left(\mathbf{T}, s, z_{2}, y_{2}\right)$. Furthermore for any random variable $\Phi$ we have the following

$$
\begin{gathered}
\left.(5.3\rangle \Phi-V_{t}^{\pi_{1}}\right)^{+} \geq\left(\Phi-V_{t}^{\pi_{2}}\right)^{+}-\mathbb{I}_{t \leq \varsigma}\left(\left|z_{2}-z_{1}\right|+\left|y_{2}-y_{1}\right|(1+\mu)\left(s+S_{t \wedge \varsigma \wedge \varrho}^{(s)}\right)\right) \\
-\mathbb{I}_{t>\varsigma}\left(\mathbb{I}_{V_{t}^{\pi}>1} \Phi+\mathbb{I}_{V_{t}^{\pi} \leq 1} V_{t}^{\pi_{1}}\right) .
\end{gathered}
$$

From (5.2)-(5.3) we get

$$
\begin{gathered}
R\left(\mathbf{T}, v, z_{2}, y_{2}\right)-R\left(\mathbf{T}, v, z_{1}, y_{1}\right) \leq \\
A+\mathcal{R}\left(\mathbf{T}, v, \pi_{2}, \sigma_{2}\right)-\mathcal{R}\left(\mathbf{T}, v, \pi_{1}, \sigma_{1}\right) \leq A+\left|z_{1}-z_{2}\right|+ \\
\sup _{\tau \in \mathcal{T}_{\mathbf{T}}} \mathbb{E}_{\mathbb{P}}\left(\left|y_{2}-y_{1}\right|(1+\mu)\left(s+S_{\tau \wedge \varsigma \curlywedge \varrho}^{(s)}\right)+\mathbb{I}_{\varsigma<\tau} \mathbb{I}_{V_{\tau}^{\pi_{1}}>1} X_{\tau}^{\mathbf{T}, v}+\mathbb{I}_{\varsigma<\tau} \mathbb{I}_{V_{\tau}^{\pi_{1}} \leq 1} V_{\tau}^{\pi_{1}}\right) \\
\leq A+\left|z_{1}-z_{2}\right|+2\left|y_{2}-y_{1}\right|(1+\mu) \mathbb{E}_{\mathbb{P}}\left(\max _{0 \leq t \leq T} S_{t}^{(s)}\right) \\
+\mathbb{E}_{\mathbb{P}}\left(\mathbb{I}_{\varsigma<\tau} \mathbb{I}_{V_{\tau}^{\pi_{1}}>1} X_{\tau}^{\mathbf{T}, v}+\mathbb{I}_{\varsigma<\tau} \mathbb{I}_{V_{\tau}^{\pi_{1}} \leq 1} V_{\tau}^{\pi_{1}}\right) .
\end{gathered}
$$

Thus in order to complete the proof it remains to show that

$$
\sup _{\tau \in \mathcal{T}_{\mathbf{T}}} \mathbb{E}_{\mathbb{P}}\left(\mathbb{I}_{\varsigma<\tau} \mathbb{I}_{V_{\tau}^{\pi_{1}}>1} X_{\tau}^{\mathbf{T}, v}+\mathbb{I}_{\zeta<\tau} \mathbb{I}_{V_{\tau}^{\pi_{1}} \leq 1} V_{\tau}^{\pi_{1}}\right)=O\left(A^{3 / 4}\right) .
$$

Let $\tau \in \mathcal{T}_{T}$. From (2.2)-(2.3) and the Hölder inequality (for $p=4, q=4 / 3$ ) it follows that there exists a constant $c=c(K)$ such that

$$
\begin{gathered}
\mathbb{E}_{\mathbb{P}}\left(\mathbb{I}_{\varsigma<\tau} \mathbb{I}_{V_{\tau}^{\pi_{1}}>1} X_{\tau}^{\mathbf{T}, v}+\mathbb{I}_{\varsigma<\tau} \mathbb{I}_{V_{\tau}^{\pi_{1}} \leq 1} V_{\tau}^{\pi_{1}}\right)= \\
\mathbb{E}_{\mathbb{Q}}\left(Z_{T}^{-1} \mathbb{I}_{\varsigma<\tau} \mathbb{I}_{V_{\tau}^{\pi_{1}}>1} X_{\tau}^{\mathbf{T}, v}+Z_{T}^{-1} \mathbb{I}_{\varsigma<\tau} \mathbb{I}_{V_{\tau}^{\pi_{1}} \leq 1} V_{\tau}^{\pi_{1}}\right) \leq \\
c\left(\left(\mathbb{E}_{\mathbb{Q}}\left[\mathbb{I}_{\varsigma<\tau} \mathbb{I}_{V_{\tau}^{\pi_{1}}>1}\right]\right)^{3 / 4}+\left(\mathbb{E}_{\mathbb{Q}}\left[\mathbb{I}_{\varsigma<\tau} \mathbb{I}_{V_{\tau}^{\pi_{1}}<1}\left(V_{\tau}^{\pi_{1}}\right)^{4 / 3}\right]\right)^{3 / 4}\right) \leq \\
c\left(\left(\mathbb{E}_{\mathbb{Q}}\left[\mathbb{I}_{\varsigma<\tau} V_{\tau}^{\pi_{1}}\right]\right)^{3 / 4}+\left(\mathbb{E}_{\mathbb{Q}}\left[\mathbb{I}_{\varsigma<\tau} V_{\tau}^{\pi_{1}}\right]\right)^{3 / 4}\right) \leq
\end{gathered}
$$

since $\left\{V_{t}^{\pi_{1}}\right\}_{t=0}^{\mathbf{T}}$ is a super-martingale with respect to $\mathbb{Q}$,

$$
\leq 2 c\left(\mathbb{E}_{\mathbb{Q}}\left[\mathbb{I}_{\varsigma<\tau} V_{\varsigma+}^{\pi_{1}}\right]\right)^{3 / 4} \leq
$$

since $V_{\varsigma+}^{\pi_{1}} \leq\left|z_{2}-z_{1}\right|+\left|y_{2}-y_{1}\right|(1+\mu)\left(s+S_{\varsigma \wedge \varrho}^{(s)}\right)$ on the event $\varsigma<\tau$,

$$
\leq 2 c\left(\left|z_{2}-z_{1}\right|+2 s\left|y_{2}-y_{1}\right|(1+\mu)\right)^{3 / 4}=O\left(A^{3 / 4}\right),
$$

and the proof is completed. 
Lemma 5.4. Let $K>0, v \in C_{++}[0, T]$ and $y \in \mathbb{R}$. Assume that $\|v\| \leq K$ and $|y| \leq \frac{\delta}{\mu} \min _{t \in[0, T]} \frac{1}{v(t)}$. Then for any $z \geq 0$ and $\mathbf{T} \in[0, T]$,

$$
R(\mathbf{T}, v, z+\delta, 0)-R(\mathbf{T}, v, z, y) \leq \hat{C}(K)|y|^{3 / 4} .
$$

Proof. Denote $s=v(T-\mathbf{T})$. Without loss of generality we assume that $y \neq 0$. Let $\left(\pi_{1}, \sigma_{1}\right) \in \mathcal{A}(\mathbf{T}, s, z, y)$ such that $R(\mathbf{T}, v, z, y)>\mathcal{R}\left(\mathbf{T}, v, \pi_{1}, \sigma_{1}\right)-|y|$. Set $\pi_{1}=$ $\left(z, y, \gamma^{(1)}\right)$. Define the stopping times $\varrho=\inf \left\{t: \gamma_{t+}^{(1)}=0\right\} \wedge \mathbf{T}$ and

$$
\varsigma=\inf \left\{t: V_{t}^{\pi_{1}} \leq|y|(1+\mu) S_{t \wedge \varrho}^{(s)}\right\} \wedge \mathbf{T} .
$$

Introduce the hedge $\left(\pi_{2}, \sigma_{2}\right) \in \mathcal{A}(\mathbf{T}, s, z+\delta, 0)$ by the relation $\sigma_{2}=\sigma_{1}$ and $\pi_{2}=$ $\left(z+\delta, 0, \gamma^{(2)}\right)$ where

$$
\gamma_{t}^{(2)}=\mathbb{I}_{t \leq \varsigma}\left(\left(\gamma_{t}^{(1)}-y\right) \mathbb{I}_{t \leq \varrho}+\gamma_{t}^{(1)} \mathbb{I}_{t>\varrho}\right) .
$$

Our assumptions imply that $g(y, s)=\delta$, and so from (2.4) we get

$$
V_{t}^{\pi_{2}}=V_{t \wedge \varsigma}^{\pi_{2}} \geq V_{t \wedge \varsigma}^{\pi_{1}}-|y|(1+\mu) S_{t \wedge \varsigma \wedge \varrho}^{(s)} \geq 0 .
$$

Thus, similarly to Lemma 5.3 we get

$$
R(\mathbf{T}, v, z+\delta, 0)-R(\mathbf{T}, v, z, y) \leq \hat{C}(K)|y|^{3 / 4},
$$

as required.

The following Proposition is the main result of this section.

Proposition 5.5. (i). The function $R:[0, T] \times C_{++}[0, T] \times \mathbb{R}_{+} \times \mathbb{R} \rightarrow \mathbb{R}$ is upper semi continuous (and hence measurable). If we restrict the function $R$ to the domain $[0, T] \times C_{++}[0, T] \times \mathbb{R}_{+} \times \mathbb{R} \backslash\{0\}$ then the function $R:[0, T] \times C_{++}[0, T] \times$ $\mathbb{R}_{+} \times \mathbb{R} \backslash\{0\} \rightarrow \mathbb{R}$ is a continuous function.

(ii). There exists a measurable function $\beta^{*}:[0, T] \times C_{++}[0, T] \times \mathbb{R}_{+} \times \mathbb{R} \rightarrow \mathbb{R}$ such that $\beta^{*}(\mathbf{T}, v, \cdot, \cdot \cdot)$ depends only on $v_{[0, T-\mathbf{T}]}$ and the infimum in $\beta$ in (3.2) is attained at $\beta^{*}=\beta^{*}(\mathbf{T}, v, z, y)$.

(iii). For any $y \in \mathbb{R}$ the function $\hat{R}_{y}:[0, T] \times C_{++}[0, T] \times\left[\delta \mathbb{I}_{y=0}, \infty\right) \rightarrow \mathbb{R}$ defined by

$$
\hat{R}_{y}(\mathbf{T}, v, z)=\hat{R}(\mathbf{T}, v, z, y)
$$

is a continuous function.

Proof. (i). Let $(\mathbf{T}, v, z, y) \in[0, T] \times C_{++}[0, T] \times \mathbb{R}_{+} \times \mathbb{R}$. Clearly for any $(\tilde{\mathbf{T}}, \tilde{v}, \tilde{z}, \tilde{y}) \in$ $[0, T] \times C_{++}[0, T] \times \mathbb{R}_{+} \times \mathbb{R}$ we have

$$
\begin{gathered}
|R(\mathbf{T}, v, z, y)-R(\tilde{\mathbf{T}}, \tilde{v}, \tilde{z}, \tilde{y})| \leq \\
\left|R(\mathbf{T}, v, z, y)-R\left(\tilde{\mathbf{T}}, v, z, \frac{y v(T-\mathbf{T})}{v(T-\tilde{\mathbf{T}})}\right)\right|+ \\
\left|R\left(\tilde{\mathbf{T}}, v, z, \frac{y v(T-\mathbf{T})}{v(T-\tilde{\mathbf{T}})}\right)-R\left(\tilde{\mathbf{T}}, \tilde{v}, z, \frac{y v(T-\mathbf{T})}{\tilde{v}(T-\tilde{\mathbf{T}})}\right)\right|+ \\
\left|R\left(\tilde{\mathbf{T}}, \tilde{v}, z, \frac{y v(T-\mathbf{T})}{\tilde{v}(T-\tilde{\mathbf{T}})}\right)-R(\tilde{\mathbf{T}}, \tilde{v}, \tilde{z}, \tilde{y})\right| .
\end{gathered}
$$

This together with Lemmas 5.1,5.2, and 5.3 yields that $R:[0, T] \times C_{++}[0, T] \times \mathbb{R}_{+} \times$ $\mathbb{R} \backslash\{0\} \rightarrow \mathbb{R}$ is a continuous function. Next, we prove that $R:[0, T] \times C_{++}[0, T] \times$ $\mathbb{R}_{+} \times \mathbb{R} \rightarrow \mathbb{R}$ is upper semi continuous. Set $s=v(T-\mathbf{T})$. For any admissible 
portfolio $\pi_{1}=\left(z, 0, \gamma^{(1)}\right)$ introduce the portfolio $\pi_{2}=\left(z, y, \gamma^{(2)}\right)$ by $\gamma_{0}^{(2)}=y$ and $\gamma_{t}^{(2)}=\gamma_{t}^{(1)}$ for $t>0$. Observe that for any $t>0$,

$$
V_{t}^{\pi_{2}}-V_{t}^{\pi_{1}}=V_{0+}^{\pi_{2}}-V_{0+}^{\pi_{1}}=g(y, s)-g\left(y-\gamma_{0+}^{(1)}, s\right)+g\left(\gamma_{0+}^{(1)}, s\right) \geq 0 .
$$

Thus $R(\mathbf{T}, v, z, y) \leq R(\mathbf{T}, v, z, 0)$. This together with (5.4) and Lemmas 5.1, 5.2 and (5.3) completes the proof.

(ii). Fix $(\mathbf{T}, v, z, y) \in[0, T] \times C_{++}[0, T] \times \mathbb{R}_{+} \times \mathbb{R}$. Set $s=v(T-\mathbf{T})$. Assume that $\Gamma(s, z, y) \neq \emptyset$. We want to show that the minimum in (3.2) is attained. Thus, let $\left\{\beta_{n}\right\}_{n=1}^{\infty} \subset \Gamma(s, z, y)$ for which

$$
\lim _{n \rightarrow \infty} R\left(\mathbf{T}, v, h\left(s, z, y, \beta_{n}\right), y+\beta_{n}\right)=\inf _{\beta \in \Gamma(s, z, y)} R(\mathbf{T}, v, h(s, z, y, \beta), y+\beta) .
$$

The set $\Gamma(s, z, y)$ is compact, and so without loss of generality we assume (by passing to a sub sequence) that the sequence $\left\{\beta_{n}\right\}_{n=1}^{\infty} \subset \Gamma(s, z, y)$ converges, thus let $\lim _{n \rightarrow \infty} \beta_{n}=\hat{\beta} \in \Gamma(s, z, y)$. First assume that $\hat{\beta} \neq-y$, then

$$
h(s, z, y, \hat{\beta})=\lim _{n \rightarrow \infty} h\left(s, z, y, \beta_{n}\right) .
$$

Thus from (5.5) and the fact that $R:[0, T] \times C_{++}[0, T] \times \mathbb{R}_{+} \times \mathbb{R} \backslash\{0\} \rightarrow \mathbb{R}$ is continuous we conclude that

$$
R(\mathbf{T}, v, h(s, z, y, \hat{\beta}), y+\hat{\beta})=\inf _{\beta \in \Gamma(s, z, y)} R(\mathbf{T}, v, h(s, z, y, \beta), y+\beta) .
$$

Next, we deal with the case $\hat{\beta}=-y$. In this case $h(s, z, y, \hat{\beta})=\delta+\lim _{n \rightarrow \infty} h\left(s, z, y, \beta_{n}\right)$, and so from (5.5) and Lemma 5.4 we obtain

$$
R(\mathbf{T}, v, h(s, z, y, \hat{\beta}), 0)=\inf _{\beta \in \Gamma(s, z, y)} R(\mathbf{T}, v, h(s, z, y, \beta), y+\beta) .
$$

We conclude that the infimum in (3.2) is attained at $\hat{\beta} \in \Gamma(s, z, y)$. It follows that there exists a measurable map $\beta^{*}:[0, T] \times C_{++}[0, T] \times \mathbb{R}_{+} \times \mathbb{R} \rightarrow \mathbb{R}$ such that $\beta^{*}=\beta^{*}(\mathbf{T}, v, z, y)$ depends only on $v_{[0, T-\mathbf{T}]}$ and the infimum in (3.2) is attained at $\beta^{*}$ provided that $\Gamma(v(T-\mathbf{T}), z, y) \neq \emptyset$. For instance, $\beta^{*}(\mathbf{T}, v, z, y)=0$ if $y=0$ and $z<\delta($ i.e. $\Gamma(v(T-\mathbf{T}), z, y)=\emptyset)$, and if $\Gamma(v(T-\mathbf{T}), z, y) \neq \emptyset$,

$$
\begin{gathered}
\beta^{*}(\mathbf{T}, v, z, y)=\min _{\tilde{\beta} \in \Gamma(v(T-\mathbf{T}), z, y)} R(\mathbf{T}, v, h(s, z, y, \tilde{\beta}), y+\tilde{\beta})= \\
\inf _{\beta \in \Gamma(s, z, y)} R(\mathbf{T}, v, h(s, z, y, \beta), y+\beta) .
\end{gathered}
$$

(iii). Fix $y \in \mathbb{R}$. Choose a sequence $\left\{\mathbf{T}_{n}, v_{n}, z_{n}\right\}_{n=1}^{\infty} \subset[0, T] \times C[0, T] \times\left[\delta \mathbb{I}_{y=0}, \infty\right)$ which converges to $(\mathbf{T}, v, z)$. From the continuity of $G$ it follows that

$$
X_{0}^{\mathbf{T}, v}=\lim _{n \rightarrow \infty} X_{0}^{\mathbf{T}_{n}, v_{n}}
$$

Set $s=v(T-\mathbf{T})$ and $s_{n}=v_{n}\left(T-\mathbf{T}_{n}\right), n \in \mathbb{N}$. Let $\beta_{n}=\beta^{*}\left(\mathbf{T}_{n}, v_{n}, z_{n}, y\right), n \in \mathbb{N}$. The sequence $\beta_{n}, n \in \mathbb{N}$ is bounded, and so without loss of generality by taking a subsequence we can assume that it converges. Denote $\hat{\beta}=\lim _{n \rightarrow \infty} \beta_{n}$. It is straightforward to check that $\hat{\beta} \in \Gamma(s, z, y)$. By using (5.8) and applying similar arguments as in (5.6)-(5.7) we get

$$
\begin{gathered}
\hat{R}(\mathbf{T}, v, z, y) \leq \min \left(\left(X_{0}^{\mathbf{T}, v}-z\right)^{+}, R(\mathbf{T}, v, h(s, z, y, \hat{\beta}), \hat{\beta}+y)\right) \\
\leq \liminf _{n \rightarrow \infty} \hat{R}\left(\mathbf{T}_{n}, v_{n}, z_{n}, y\right) .
\end{gathered}
$$


This yields the lower semi-continuity of $\hat{R}_{y}$. Thus, it remains to establish upper semi-continuity. Let $\tilde{\beta}=\beta^{*}(\mathbf{T}, s, z, y)$. First assume that $h(s, z, y, \tilde{\beta})>0$. Then for sufficiently large $n$, we have $\tilde{\beta} \in \Gamma\left(s_{n}, z_{n}, y\right)$ and

$$
h(s, z, y, \tilde{\beta})=\lim _{n \rightarrow \infty} h\left(s_{n}, z_{n}, y, \tilde{\beta}\right) .
$$

Thus from (5.8) and the fact that $R$ is upper semi-continuous we get

$$
\begin{aligned}
\hat{R}(\mathbf{T}, v, z, y)= & \min \left(\left(X_{0}^{\mathbf{T}, v}-z\right)^{+}, R(\mathbf{T}, v, h(s, z, y, \tilde{\beta}), \tilde{\beta}+y)\right) \\
& \geq \limsup _{n \rightarrow \infty} \hat{R}\left(\mathbf{T}_{n}, v_{n}, z_{n}, y\right) .
\end{aligned}
$$

Finally, assume that $h(s, z, y, \tilde{\beta})=0$. Let $\pi=(0, \tilde{y}, \gamma)$ be an admissible portfolio for some $\tilde{y}$. From the fact that the geometric Brownian motion can increase or decrease for any amount (with positive probability) on any time interval it follows that $\gamma_{t}=0$ for $t>0$. Indeed, for otherwise the portfolio value can become negative with positive probability. Thus

$$
R(\mathbf{T}, v, 0, \tilde{y})=\inf _{\sigma \in \mathcal{T}_{\mathbf{T}}} \sup _{\tau \in \mathcal{T}_{\mathbf{T}}} \mathbb{E}_{\mathbb{P}}\left(X_{\sigma}^{\mathbf{T}, v} \mathbb{I}_{\sigma<\tau}+Y_{\tau}^{\mathbf{T}, v} \mathbb{I}_{\tau \leq \sigma}\right) .
$$

In particular, $R(\mathbf{T}, v, 0, \tilde{y})$ does not depend on $\tilde{y}$. From (2.3) and the fact that $F, G$ are continuous it follows that $R(\cdot, \cdot \cdot, 0, \tilde{y})$ is continuous. For any $n \in \mathbb{N}$, $-y \in \Gamma\left(s_{n}, z_{n}, y\right)$, and so from the upper semi continuity of $R$ it follows that

$$
\begin{gathered}
\hat{R}(\mathbf{T}, v, z, y)=\min \left(\left(X_{0}^{\mathbf{T}, v}-z\right)^{+}, R(\mathbf{T}, v, 0, \tilde{\beta}+y)\right) \\
\geq \lim \sup _{n \rightarrow \infty} \min \left(\left(X_{0}^{\mathbf{T}_{n}, v_{n}}-z_{n}\right)^{+}, R\left(\mathbf{T}_{n}, v_{n}, z_{n}-\delta \mathbb{I}_{y=0}, 0\right)\right) \\
\geq \lim \sup _{n \rightarrow \infty} \hat{R}\left(\mathbf{T}_{n}, v_{n}, z_{n}, y\right) .
\end{gathered}
$$

From (5.9) - (5.10) we derive the upper semi continuity of $\hat{R}_{y}$, and the proof is completed.

\section{Acknowledgement}

The authors were partially supported by Einstein Foundation Grant no.A 2012 137. The first author also acknowledges support of the Marie Curie Actions fellowships Grant no.618235 and the second author of Israeli Science Foundation Grant no. $82 / 10$.

\section{REFERENCES}

[1] Altarovici, A., Muhle-Karbe, J. And Soner, H.M. (2015). Asymptotics with fixed transaction costs, Finance and Stochastics. 19, 363-414.

[2] Cvitanic, J. and Karatzas, I. (1996). Backward stochastic differential equations with reflection and Dynkin games, Annals of Probability. 24, 2024-2056.

[3] Dolinsky, Y. Limit Theorems for partial hedging under transaction Costs. (2014). Math. Finance. 24, 567-597.

[4] Dolinsky, Y. (2013). Hedging of game Options with the presence of transaction costs. Ann. Appl. Probab. 23, 2212-2237.

[5] Dolinsky, Y. AND Kifer, Y. (2007). Hedging with risk for game options in discrete time. Stochastics. 79, 169-195.

[6] Dolinsky, Y. and Kifer, Y. (2008). Binomial approximations of shortfall risk for game options. Ann. Appl. Probab. 18, 1737-1770.

[7] Eastham, J.F. and Hastings, K.J. (1988). Optimal impulse control of portfolios. Math.Oper.Res. 13, 588-605. 
[8] Guasoni, P. (2002). Risk minimization under transaction costs. Finance and Stochastics. 6, 91-113.

[9] Guasoni, P. (2002). Optimal investment with transaction costs and without semimartingales. Ann.Appl.Probab. 12, 1227-1246.

[10] Iron, Y. AND KifER, Y. Hedging of swing game options in continuous time. Stochastics. 83, 365-404.

[11] Kamizono, K. (2001). Partial hedging under proportional transaction costs. PhD dissertation.

[12] Kamizono, K. (2003). Partial hedging under transaction costs. SIAM J.Control Optimization. 5, 1545-1558.

[13] Kifer, Y. (2006). Error estimates for binomial approximations of game options. Ann.Appl.Probab. 16, 984-1033.

[14] Kifer, Y. (2000). Game options. Finance and Stoch. 4, 443-463.

[15] Kifer, Y. (2013). Dynkin games and Israeli options. ISRN Probability and Statistics. Id. 856458 .

[16] Korn, R. (1998). Portfolio optimisation with strictly positive transaction costs and impulse control. Finance and Stochastics.2, 85-114.

[17] Kobylanski, M. And Quenez, M.C. (2012). Optimal stopping time problem in a general framework. Electron. J. Probab. 17, 1-28.

[18] Lepeltier, J.P. and Maingueneau, J.P. (1984). Le jeu de Dynkin en theorie generale sans lhypothese de Mokobodski. Stochastics. 13, 24-44.

[19] Lo, A.W., Mamaysky, H. And Wang, J. (2004). Asset prices and trading volume under fixed transaction costs. J. Polit. Econ. 112, 1054-1090.

[20] Morton, A.J. and Pliska, S.R. (1995). Optimal portfolio management with fixed transaction costs. Math. Finance 5, 337-356.

[21] B.Oksendal and A.Sulem, Oksendal, B. And Sulem, A. (2002). Optimal consumption and portfolio with both fixed and proportional transaction costs. SIAM J.Control Optimization. 6, 1765-1790.

Department of Statistics, Hebrew University of Jerusalem, IsRael, E.mail: yan.yolinsky@Mail.huji.aC.IL

Department of Mathematics, Hebrew University of Jerusalem, Israel, e.mail: Kifer@math.huji.aC.IL 